\author{
Biogeografía
}

\title{
Diversidad y distribución geográfica de los mamíferos terrestres del estado de Oaxaca, México
}

\author{
Diversity and geographical distribution of the terrestrial mammals of the state of Oaxaca, Mexico \\ Miguel Briones-Salas*, Malinalli Cortés-Marcial y Mario C. Lavariega \\ Centro Interdisciplinario de Investigación para el Desarrollo Integral Regional, Unidad Oaxaca, Instituto Politécnico Nacional, Calle Hornos 1003, Santa Cruz \\ Xoxocotlán, 71230, Oaxaca, México \\ Recibido el 22 de noviembre de 2014; aceptado el 5 de febrero de 2015 \\ Disponible en Internet el 29 de agosto de 2015
}

\begin{abstract}
Resumen
En este trabajo se presenta una lista actualizada de las especies de mamíferos terrestres de Oaxaca. Se analizó la riqueza acumulada, los patrones espaciales de riqueza, endemismo, estado de conservación y la diversidad beta taxonómica. La información proviene de recolectas recientes y se complementa con los registros de ejemplares depositados en colecciones científicas y de literatura especializada. Los resultados obtenidos muestran que a partir de la década de 1960 se incrementó el esfuerzo de recolecta; actualmente se conocen 28,060 registros. La composición taxonómica en Oaxaca está representada por 216 especies, incluyendo 48 monotípicas y 168 politípicas con 209 subespecies; esto suma un total de 257 taxones. La mayor riqueza de especies se registró en las subprovincias fisiográficas: Sierra Madre de Oaxaca (154), Planicie Costera de Tehuantepec (135) y Sierra Madre del Sur (127). La baja similitud taxonómica entre subprovincias (0.36-0.82) explica la elevada diversidad de mamíferos en Oaxaca. Derechos Reservados (C) 2015 Universidad Nacional Autónoma de México, Instituto de Biología. Este es un artículo de acceso abierto distribuido bajo los términos de la Licencia Creative Commons CC BY-NC-ND 4.0.
\end{abstract}

Palabras clave: Conservación; Diversidad beta taxonómica; Endemismo; Riqueza acumulada; Subprovincias fisiográficas

\section{Abstract}

In this study we present an updated taxonomic list of the terrestrial mammals of Oaxaca. The accumulated species richness, spatial richness patterns, endemicity, conservation status and taxonomic beta diversity was analyzed. We considered specimens deposited in mammal collections, and specialized literature. The results show that from the 1960's the collection effort increased, until the 28,060 records were obtained. The taxonomic composition of terrestrial mammals in Oaxaca is represented by 216 species, including 48 monotypic and 168 polytypic with 209 subspecies, a total of 257 taxa. The highest species richness occurs in the Sierra Madre de Oaxaca (154) and the Coastal Plain of Tehuantepec (135) and Sierra Madre del Sur (127). The low taxonomic similarity between subprovinces (0.36 to 0.82 ) explains the great diversity of mammals in Oaxaca.

All Rights Reserved (C) 2015 Universidad Nacional Autónoma de México, Instituto de Biología. This is an open access item distributed under the Creative Commons CC License BY-NC-ND 4.0.

Keywords: Conservation; Taxonomic beta diversity; Endemism; Species richness accumulation; Physiographic subprovinces

\section{Introducción}

El estado de Oaxaca, al sur de México, presenta una gran diversidad fisiográfica, climática, florística y faunística, y se

\footnotetext{
* Autor para correspondencia.

Correo electrónico: mbriones@ipn.mx (M. Briones-Salas).

La revisión por pares es responsabilidad de la Universidad Nacional Autónoma de México.
}

encuentra entre las 3 entidades con mayor riqueza de especies de artrópodos, plantas vasculares y vertebrados, razones por las que se le ha considerado como un estado megadiverso (García-Mendoza, 2004; Llorente y Ocegueda, 2008). El conocimiento de la riqueza de especies de mamíferos terrestres de Oaxaca ha variado como reflejo de nuevos estudios taxonómicos y de sistemática, y por el incremento de nuevas exploraciones. Goodwin (1969) fue el primer autor en analizar la taxonomía, distribución geográfica y ecología de los mamíferos de Oaxaca 
y aportó información para 274 especies y subespecies; posteriormente, Ramírez-Pulido, Briton, Perdomo y Castro (1986) revisaron la taxonomía y sistemática de los mamíferos mexicanos y enlistan 264 taxones para Oaxaca; Briones-Salas y Sánchez-Cordero (2004) revisan bases de datos de colecciones científicas, recolectas y publicaciones, y enlistan un total de 261 taxones; recientemente, Llorente y Ocegueda (2008) registran 194 especies de mamíferos para el estado y Sánchez-Cordero et al. (2014) reconocen para Oaxaca 191 de las 564 especies que existen en México.

A pesar de su gran riqueza, en Oaxaca se presentan graves problemas para la conservación de su fauna, lo que se ha reflejado en la pérdida de su diversidad, a consecuencia de diversos factores como la explosión demográfica, la destrucción o modificación de sus hábitats, los incendios forestales, el cambio de uso de suelo para actividades agropecuarias, el aprovechamiento forestal clandestino, uso de plaguicidas, cacería sin control y saqueo, entre otros más (González-Pérez, Briones-Salas y Alfaro, 2004). Es evidente que si no se tiene el conocimiento de la existencia y distribución de los mamíferos del estado, difícilmente se podrán sugerir estrategias de conservación para estas especies. Por lo tanto, el objetivo del presente estudio es actualizar la lista de especies de mamíferos de Oaxaca, presentar un análisis histórico del conocimiento de la mastofauna y determinar su situación de conservación, distribución geográfica por subprovincia fisiográfica y altitudinal, así como analizar la riqueza de especies y la diversidad beta taxonómica, de manera que sirva de insumo para su conservación y de base para estudios futuros con enfoques ecológicos, de manejo o aprovechamiento.

\section{Materiales y métodos}

El estado de Oaxaca se localiza al sur de México, entre las coordenadas geográficas $15^{\circ} 39^{\prime}$ y $18^{\circ} 39^{\prime} \mathrm{N}$ y $93^{\circ} 52^{\prime}$ y $98^{\circ} 32^{\prime}$ $\mathrm{O}$, con una superficie de $95,364 \mathrm{~km}^{2}$, que representa el $4.8 \%$ del territorio nacional (García-Mendoza, 2004). Tiene una orografía compleja y se reconocen 12 subprovincias fisiográficas que se distinguen por poseer rasgos geomorfológicos estructurales propios (Ortiz-Pérez, Hernández-Santana y Figueroa Mah-Eng, 2004). El estado presenta un intervalo altitudinal que va desde el nivel del mar hasta los 3,600 m, lo que facilita la presencia de 26 tipos de climas, desde cálidos secos en la Planicie Costera del Pacífico hasta templados en los picos montañosos de las principales sierras (Trejo, 2004). Existen 26 tipos de comunidades vegetales, de las cuales el bosque de pino, la selva baja caducifolia, pastizales y selva alta perennifolia cubren el 20,13, 12 y $11 \%$ de la superficie del estado, respectivamente (Torres-Colín, 2004).

Se partió de una base de datos que contiene información de ejemplares depositados en 28 colecciones nacionales e internacionales y documentados en literatura científica (Briones-Salas y Sánchez-Cordero, 2004), la cual fue actualizada hasta julio de 2014. Se consideraron las recolectas realizadas en los últimos 8 años por el equipo de trabajo de los autores. Los ejemplares recolectados están depositados en la colección de mamíferos del Centro Interdisciplinario de Investigación para el Desarrollo Integral Regional, Unidad Oaxaca, del Instituto
Politécnico Nacional, con registro por parte de la Secretaría de Medio Ambiente y Recursos Naturales (OAX.MA.026.0497) y se incluyeron en la base de datos general.

Para el análisis de datos, se realizaron curvas de acumulación simples de registros y especies en intervalos de 10 años a partir de los primeros registros formales de recolecta (1890). Se elaboró una lista de especies y subespecies que fue actualizada en su nomenclatura y arreglo sistemático de acuerdo con RamírezPulido, González-Ruíz, Gardner y Arroyo-Cabrales (2014). Se consultó la distribución geográfica de las especies y subespecies en los trabajos de Hall (1981), Ceballos y Oliva (2005) y Carraway (2007) para identificar taxones endémicos a México y Oaxaca. Para determinar la situación de conservación y protección se consultó la lista roja de la Unión Internacional para la Conservación de la Naturaleza (IUCN, 2014), los apéndices de la Convención sobre el Comercio Internacional de Especies Amenazadas de Fauna y Flora Silvestres (CITES, 2011) y la Norma Oficial Mexicana 059 (Nom-059- Semarnat-2010; Semarnat, 2010).

Se obtuvo la riqueza de especies, géneros, familias y órdenes a nivel estatal, altitudinal y por subprovincia fisiográfica (Ortiz-Pérez et al., 2004). La similitud específica entre las subprovincias se obtuvo con el índice de Jaccard, con el método de pares asociados al azar sin peso aritmético (UPGMA, por sus siglas en inglés), y la diversidad beta taxonómica a nivel supraespecífica entre subprovincias se evaluó con el índice de Izsák y Price (2001) modificado por Bacaro, Ricotta y Mazzoleni (2007).

\section{Resultados}

La base de datos de mamíferos terrestres para el estado de Oaxaca estuvo conformada por 32,829 registros, de los cuales 4,770 corresponden a ejemplares albergados en colecciones científicas y que fueron documentados en una o más publicaciones (Goodwin, 1969 y Hall, 1981); por tanto, el número de registros únicos es de 28,060, de los cuales 3,308 están presentes en literatura y 24,752 se encuentran en 23 colecciones extranjeras y 4 nacionales: 14,348 y 10,404 registros, respectivamente. Las colecciones que cuentan con mayor número de ejemplares son el American Museum of Natural History, New York (5,666 registros), la colección mastozoológica del Centro Interdisciplinario de Investigación para el Desarrollo Integral Regional, Unidad Oaxaca, del Instituto Politécnico Nacional (5,062 registros) y la colección nacional de mamíferos, Instituto de Biología, Universidad Nacional Autónoma de México (3,370 registros) (tabla 1). Del total de registros 24,406 (86.97\%) cuentan con nombre de recolector. M. Briones-Salas es el recolector con mayor número de registros $(3,490)$, seguido por T. MacDougall $(3,222)$, J. Martínez-Vázquez (1,271), W. J. Schaldach (987) y M. D. Tuttle (936).

Los esfuerzos formales para documentar la mastofauna de Oaxaca se presentaron en la década de 1890 con A. C. Buller, quien recolectó los primeros mamíferos en la Planicie Costera de Tehuantepec y están depositados en The Field Museum of Natural History, Chicago. A partir de la década de 1960, se incrementa el esfuerzo de muestreo con las exploraciones de $\mathrm{T}$. 
Tabla 1

Número de ejemplares de mamíferos terrestres de Oaxaca depositados en colecciones científicas nacionales y extranjeras.

\begin{tabular}{|c|c|c|}
\hline Siglas de la colección & Nombre de la colección & Número de registros \\
\hline AMNH & American Museum of Natural History, New York & 5,666 \\
\hline OAXMA & Colección Regional Mastozoológica, CIIDIR-IPN Unidad Oaxaca & 5,062 \\
\hline CNMA & Colección Nacional de Mamíferos, Instituto de Biología, UNAM & 3,370 \\
\hline KU & University of Kansas, Museum of Natural History & 2,468 \\
\hline LACM & Natural History Museum of Los Angeles County & 1,613 \\
\hline TCWC & $\begin{array}{l}\text { Texas A \& M University, Texas Cooperative Wildlife Collection, Wildlife \& } \\
\text { Fisheries Sciences Department }\end{array}$ & 1,179 \\
\hline ENCB & Escuela Nacional de Ciencias Biológicas, Instituto Politécnico Nacional & 1,166 \\
\hline CAS & California Academy of Sciences & 994 \\
\hline MZFC & Museo de Zoología Alfonso L. Herrera, Facultad de Ciencias, UNAM & 806 \\
\hline TTU & Texas Tech University, Museum of Texas Tech University & 606 \\
\hline UMMZ & University of Michigan, Museum of Zoology & 556 \\
\hline FMNH & The Field Museum & 331 \\
\hline USNM & United States National Museum of Natural History & 205 \\
\hline MSB & University of New Mexico, Museum of Southwestern Biology & 193 \\
\hline MVZ & University of California, Berkeley, Museum of Vertebrate Zoology & 151 \\
\hline FSM & University of Florida, Florida Museum of Natural History & 86 \\
\hline UM & University of Michigan, Zoology Museum, Ann Arbor & 67 \\
\hline LSUMZ & Louisiana State University, Museum of Natural Science & 61 \\
\hline $\mathrm{CM}$ & Carnegie Museum of Natural History & 44 \\
\hline $\mathrm{MCZ}$ & Harvard University, Museum of Comparative Zoology & 41 \\
\hline UWBM & $\begin{array}{l}\text { University of Washington, Thomas Burke Memorial Washington State } \\
\text { Museum }\end{array}$ & 37 \\
\hline ROM & Royal Ontario Museum & 33 \\
\hline BM & British Museum (Natural History) London & 4 \\
\hline MMNH & University of Minnesota, James Ford Bell Museum of Natural History & 4 \\
\hline SDNHM & San Diego Natural History Museum & 4 \\
\hline MSU & Michigan State University, Lansing & 3 \\
\hline $\mathrm{CC}$ & Richard S. Crossin Collection, Tucson, Arizona & 1 \\
\hline UTEP & University of Texas at El Paso, Centennial Museum & 1 \\
\hline
\end{tabular}

MacDougall, S. Booth, J. Schaldach y M. D. Tuttle, entre otros, quienes recolectaron en gran parte del estado y adicionaron un gran número de especies (188) (fig. 1). La curva acumulada de registros muestra que a partir de 1940 se intensificaron las exploraciones mastozoológicas en Oaxaca con 2,148 y alcanzaron los 16,489 registros en 1980; posteriormente, el incremento fue constante hasta el 2014. En cuanto al número acumulado de especies, en la década de 1910 se conocían 72 especies, cifra

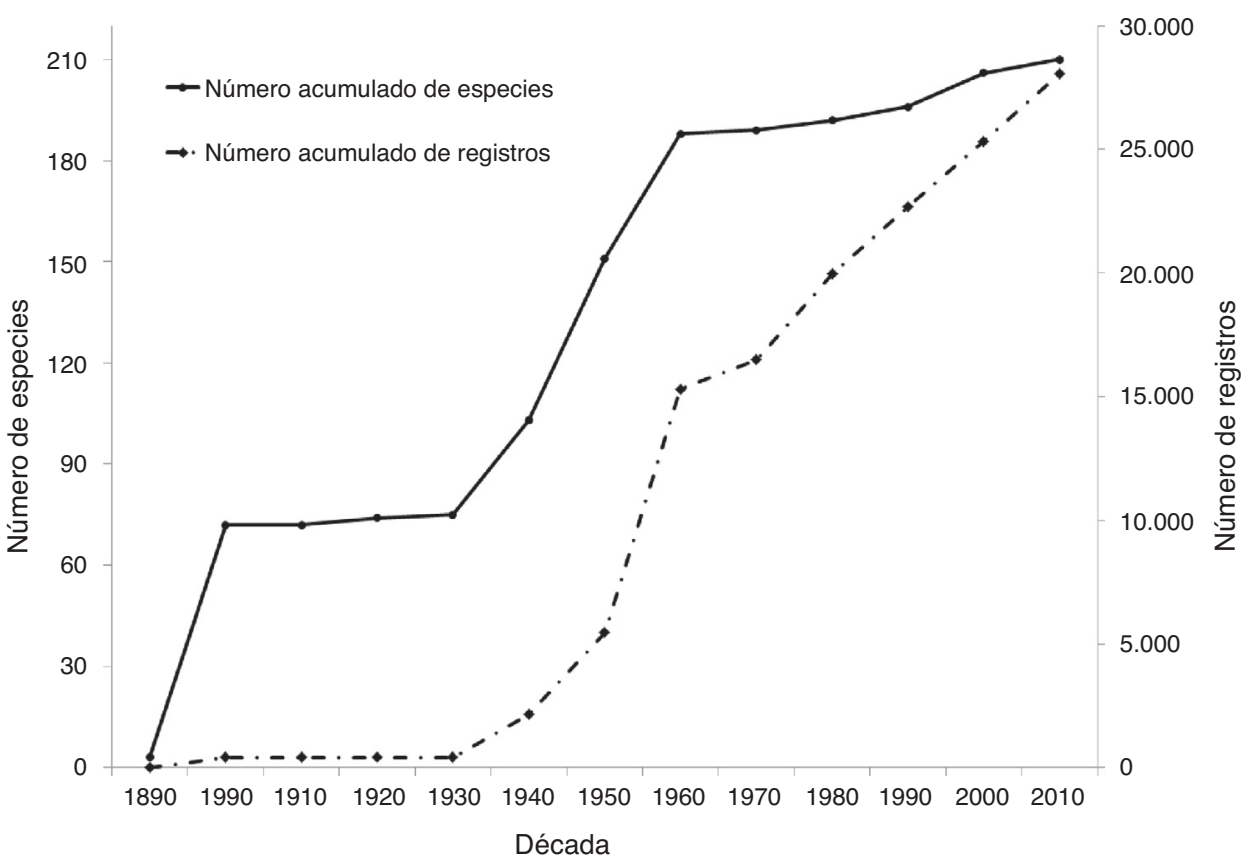

Figura 1. Número de especies y especies acumuladas de mamíferos terrestres en el estado de Oaxaca a partir de la primera década de recolecta. 
que se mantuvo casi constante hasta 1940, posteriormente se incrementa a 189 en 1970, década a partir de la cual la adición de nuevas especies es menor (fig. 1).

La lista de especies de mamíferos terrestres registrados en Oaxaca está compuesta por 216 especies, incluyendo 48 monotípicas y 168 politípicas con 209 subespecies, lo que suma un total de 257 taxones que pertenecen a 118 géneros, 29 familias y 11 órdenes (anexo), lo que equivale a 43.54, 70.23, 82.85 y $91.66 \%$ de las especies, géneros, familias y órdenes presentes en México (Ramírez-Pulido et al., 2014). Del total de especies, 120 $(55.56 \%)$ pertenecen a mamíferos no voladores, mientras que $96(44.44 \%)$ son voladores (orden Chiroptera). El orden Rodentia es el segundo en mayor representatividad, con 64 especies (29.63\%). Los órdenes menos representados fueron Cingulata y Perissodactyla con solo una especie ( $0.46 \%$ cada uno) (tabla 2$)$. Las familias representadas por el mayor número de especies son Cricetidae, Phyllostomidae y Vespertilionidae, con 49 (22.68\%), $45(20.83 \%)$ y $23(10.64 \%)$ especies, respectivamente; mientras que 8 familias están representadas por una sola especie (e.g., Myrmecophagidae, Tapiridae, Cyclopedidae; tabla 2).

Los órdenes con mayor número de registros son Rodentia con 13,258 registros (47\%) y Chiroptera con $11,876(42 \%)$. El orden Pilosa solo estuvo representado por 55 registros $(0.2 \%)$ (tabla 2). Las familias con mayor número de registros son Cricetidae con 10,953 registros (39\%), seguida por Phyllostomidae con 8,265 (29\%) y Heteromyidae con $1,591(6 \%)$. La familia con menor representatividad es Cyclopedidae con solo 3 registros, los cuales representan el $0.01 \%$ del total.

Oaxaca cuenta con 49 especies endémicas de México, de las cuales 14 se distribuyen exclusivamente en la entidad; estas cifras representan el 22.79 y $6.51 \%$ del total de especies, respectivamente. La mayor presencia de especies endémicas en Oaxaca se registró en la Sierra Madre de Oaxaca con 10 especies (e.g., Microtus oaxacensis, Habromys ixtlani y Habromys chinanteco), la Planicie Costera del Istmo de Tehuantepec con 3 (e.g., Lepus flavigularis, Orthogeomys cuniculus) y los Valles Centrales con 3 (e.g., Cryptotis peregrina, C. phillipsii) (anexo).

Del total de especies, 32 se encuentran en alguna categoría de riesgo de la lista roja de la IUCN, 10 especies están en los apéndices de la CITES y 61 especies están enlistadas en la Nom-059. El número de especies en cada categoría de la IUCN son: 4 en peligro crítico, 13 en peligro, 6 vulnerables y 7 casi amenazadas. La CITES enlista a 7 especies en el Apéndice I y 3 en el Apéndice II. En cuanto a la Nom-059-Semarnat-2010, 13 están en peligro de extinción, 26 se encuentran amenazadas y 21 están sujetas a protección especial. Canis lupus baileyi se

Tabla 2

Número de registros por categoría taxonómica de mamíferos terrestres de Oaxaca.

\begin{tabular}{|c|c|c|c|c|}
\hline Orden & Familia & Número de registros & Porcentaje & Número de especies \\
\hline \multirow[t]{6}{*}{ Rodentia } & Cricetidae & 10,953 & 39.04 & 48 \\
\hline & Heteromyidae & 1,591 & 5.67 & 5 \\
\hline & Sciuridae & 440 & 1.57 & 4 \\
\hline & Cuniculidae & 63 & 0.22 & 1 \\
\hline & Erethizontidae & 38 & 0.14 & 1 \\
\hline & Dasyproctidae & 13 & 0.05 & 1 \\
\hline & Emballonuridae & 1,307 & 4.66 & 8 \\
\hline & Mormoopidae & 982 & 3.50 & 4 \\
\hline & Vespertilionidae & 718 & 2.56 & 23 \\
\hline & Molossidae & 407 & 1.45 & 13 \\
\hline & Natalidae & 147 & 0.52 & 1 \\
\hline & Noctilionidae & 49 & 0.17 & 1 \\
\hline & Canidae & 163 & 0.58 & 3 \\
\hline & Mustelidae & 100 & 0.36 & 4 \\
\hline Total Carnivora & & 1,068 & 3.81 & 23 \\
\hline Soricomorpha & Soricidae & 566 & 2.02 & 11 \\
\hline Didelphimorphia & Didelphidae & 403 & 1.44 & 7 \\
\hline \multirow[t]{2}{*}{ Artiodactyla } & Tayassuidae & 201 & 0.72 & 2 \\
\hline & Cervidae & 180 & 0.64 & 2 \\
\hline Total Artiodactyla & & 381 & 1.36 & 4 \\
\hline Lagomorpha & Leporidae & 198 & 0.71 & 5 \\
\hline Perissodactyla & Tapiridae & 105 & 0.37 & 1 \\
\hline Cingulata & Dasypodidae & 84 & 0.30 & 1 \\
\hline Primates & Atelidae & 66 & 0.24 & 2 \\
\hline Pilosa & Myrmecophagidae & 52 & 0.19 & 1 \\
\hline
\end{tabular}


Tabla 3

Representatividad taxonómica de mamíferos terrestres en las subprovincias fisiográficas de Oaxaca.

\begin{tabular}{|c|c|c|c|c|c|}
\hline Subprovincia fisiográfica & Número de registros & Órdenes & Familias & Géneros & Especies \\
\hline Sierra Madre de Oaxaca & 9,520 & 11 & 28 & 93 & 154 \\
\hline Planicie Costera de Tehuantepec & 5,284 & 11 & 29 & 87 & 135 \\
\hline Sierra Madre del Sur & 4,279 & 10 & 24 & 73 & 127 \\
\hline Planicie Costera del Golfo & 3,039 & 11 & 27 & 73 & 101 \\
\hline Planicie Costera del Pacífico & 2,490 & 11 & 27 & 74 & 120 \\
\hline Montañas y Valles del Centro & 2,121 & 9 & 25 & 62 & 93 \\
\hline Fosa de Tehuacán & 1,409 & 6 & 16 & 47 & 64 \\
\hline Sierra Madre del Sur de Oaxaca y Chiapas & 1,364 & 11 & 26 & 77 & 105 \\
\hline Valles Centrales & 1,017 & 10 & 26 & 70 & 122 \\
\hline Montañas y Valles del Occidente & 1,011 & 8 & 20 & 64 & 15 \\
\hline Depresión del Istmo de Tehuantepec & 806 & 8 & 20 & 48 & 64 \\
\hline Depresión del Balsas & 3 & 2 & 2 & 2 & 2 \\
\hline
\end{tabular}

encuentra probablemente extinto de medio silvestre por la IUCN y la Nom-059-Semarnat-2010 (anexo).

Recientemente la Secretaría de Medio Ambiente y Recursos Naturales (Semarnat) publicó en el Diario Oficial de la Federación un listado de especies que ofrecen oportunidades para dar mayor alcance a los esfuerzos de conservación (Semarnat, 2014). Dentro de este listado se encuentran 13 especies en el estado de Oaxaca (anexo).

Las subprovincias con la mayor cantidad de registros y más estudiadas son, en orden decreciente: la Sierra Madre de Oaxaca con 9,520 registros (29.43\%), la Planicie Costera de Tehuantepec con 5,285 (16.34\%) y la Sierra Madre del Sur con 4,279 (13.23\%). En cambio, la Depresión del Balsas se encuentra poco estudiada, ya que solo cuenta con 3 registros (tabla 3; fig. 2). El número de especies de mamíferos varió notoriamente en cada una de las subprovincias fisiográficas, desde 154 en la Sierra Madre de Oaxaca hasta 2 en la Depresión del Balsas (tabla 3). En general, la Sierra Madre de Oaxaca, Planicie Costera de Tehuantepec y la Sierra Madre del Sur son las subprovincias que cuentan con la mayor cantidad de especies endémicas y de especies en

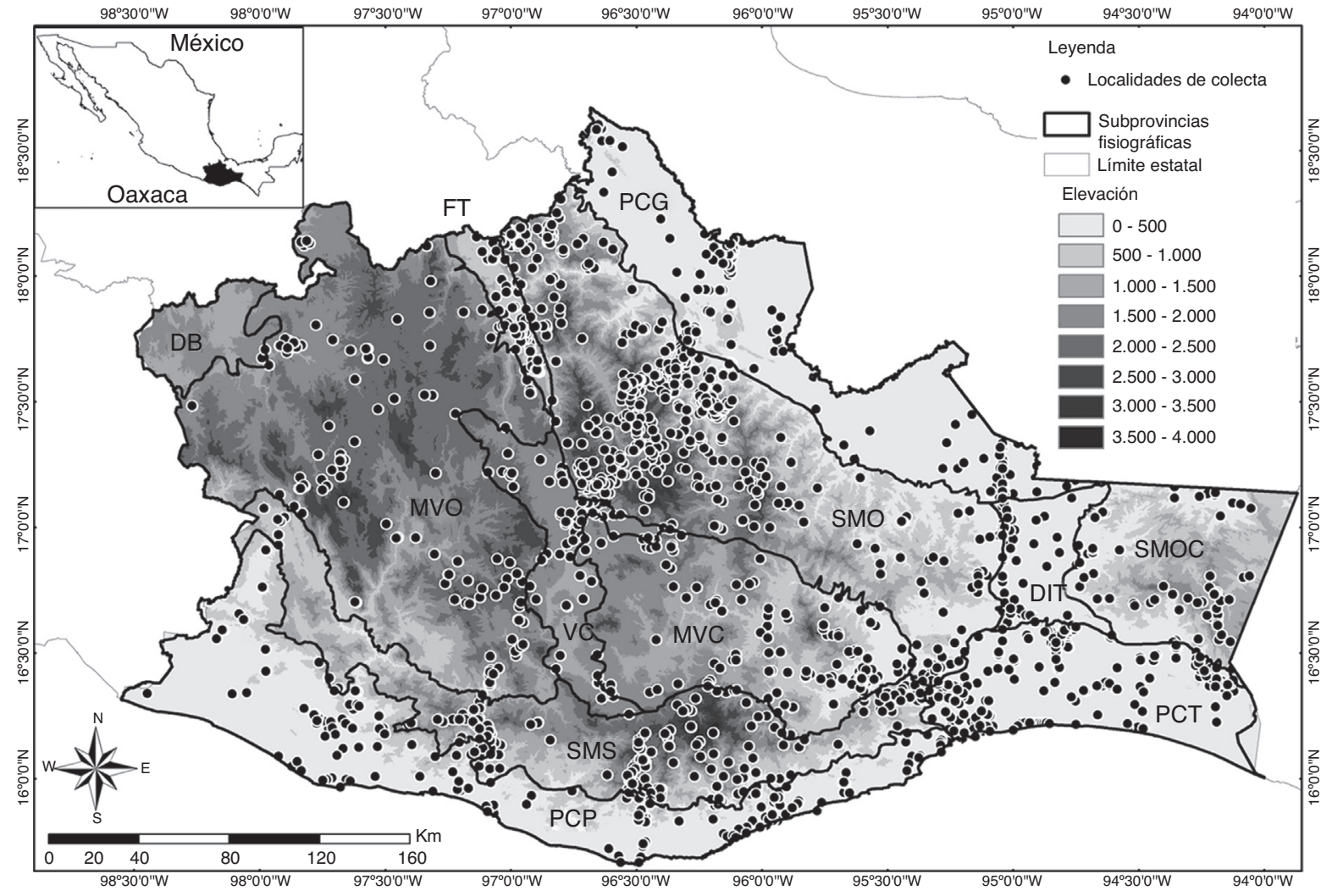

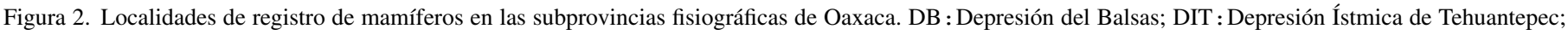

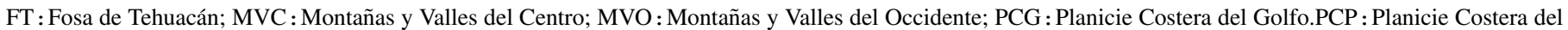

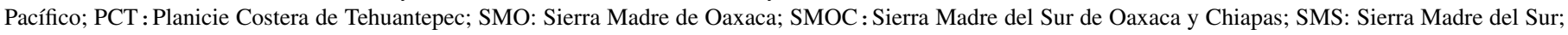
VC: Valles Centrales. 
alguna categoría de riesgo. Por el contrario, las subprovincias que cuentan con la menor cantidad de especies con estas características son la Planicie Costera del Golfo, Fosa de Tehuacán y Depresión del Balsas.

Con un coeficiente de correlación de 0.967 , en el análisis de similitud de Jaccard se reconocen 2 principales grupos: el formado por las subprovincias que se encuentran en la vertiente del Atlántico (Planicie Costera del Golfo, Sierra Madre del Sur de Oaxaca y Chiapas, y la Depresión del Istmo de Tehuantepec) y el que integran las subprovincias del centro de Oaxaca (Sierra Madre de Oaxaca, Sierra Madre del Sur, Montañas y Valles del Centro, Montañas y Valles del Occidente) y de la vertiente del Pacífico (Planicie Costera del Pacífico y Planicie Costera de Tehuantepec). La mayor similitud taxonómica específica entre subprovincias se presenta entre la Planicie Costera de Pacífico y la Sierra Madre del Sur (78\%), entre la Planicie Costera de Tehuantepec y la Planicie Costera del Pacífico (68\%), entre la Sierra Madre de Oaxaca y la Sierra Madre del Sur (64\%) y entre la Planicie Costera del Pacífico y la Sierra Madre de Oaxaca (64\%). En cambio, en las subprovincias Sierra Madre del Sur de Oaxaca y Chiapas y los Valles Centrales (28\%), la Depresión del Istmo de Tehuantepec y las Montañas y Valles del Occidente $(28 \%)$ y la Fosa de Tehuacán y la Sierra Madre de Oaxaca y Chiapas fueron las de menor similitud (fig. 3). Al incluir categorías taxonómicas supraespecíficas, la mayor similitud entre subprovincias se observó entre Sierra Madre del Sur y la Planicie Costera del Pacífico (81\%), la Planicie Costera Tehuantepec y la Planicie Costera del Pacífico (75\%), así como la Sierra Madre de Oaxaca y la Sierra Madre del Sur (69\%). En cambio, las subprovincias más disímiles fueron la Depresión del Istmo de Tehuantepec y las Montañas y Valles del Occidente (0.36),

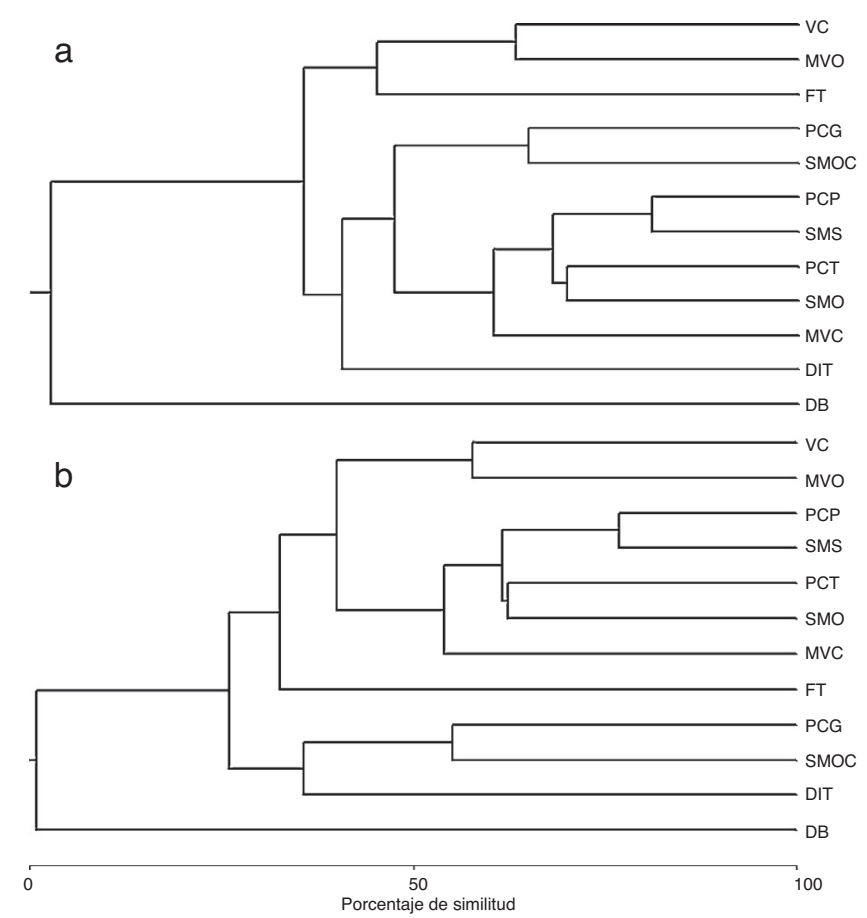

Figura 3. Análisis de similitud mastofaunística de las subprovincias fisiografías de Oaxaca. la Planicie Costera del Golfo y los Valles Centrales (36\%), así como la Sierra Madre de Oaxaca y Chiapas y los Valles Centrales (38\%) (fig. 3).

\section{Discusión}

La colección con más ejemplares recolectados de México es la Colección Nacional de Mamíferos, seguida por la Universidad de Kansas y las Escuela Nacional de Ciencias, mientras que el American Museum of Natural History ocupa el séptimo lugar (Lorenzo et al., 2012). La colección científica con la mayor cantidad de ejemplares recolectados en Oaxaca es la Colección de Mamíferos del Centro Interdisciplinario de Investigación para el Desarrollo Integral Regional, Unidad Oaxaca, del Instituto Politécnico Nacional -Oaxaca, seguida por el American Museum of Natural History y la Colección Nacional de Mamíferos. En ambos casos es relevante que instituciones nacionales tengan una alta representatividad de ejemplares de mamíferos en México, lo que facilita su consulta. Guevara-Chumacero, López-Wilchis y Sánchez-Cordero (2001) revisaron publicaciones sobre los mamíferos de México entre 1890 y 1995 y encontraron que hubo un incremento notable a partir de la década de los cincuenta, como reflejo de una mayor cantidad de científicos e instituciones interesados en los mamíferos. Este patrón fue observado en la cantidad de recolectas de mamíferos y registro de especies acumuladas de Oaxaca; asimismo, en dicho estudio y en el presente, se observó un relevo en la nacionalidad de autores y recolectores, respectivamente, a partir de la década de los ochenta con el aumento de mastozoólogos mexicanos realizando exploraciones (Briones-Salas, Cortés-Marcial y Bonilla, 2006; Sosa-Escalante, Hernández-Betancourt, PechCanche, MacSwinney y Díaz-Gamboa, 2014). Debido a lo antes mencionado, la fauna de mamíferos terrestres de Oaxaca está representada por 216 especies y 257 taxones, y la ubica como la entidad federativa con mayor diversidad de mamíferos en México, seguida por Chiapas y Veracruz (Llorente y Ocegueda, 2008; Retana y Lorenzo, 2002; Sánchez-Cordero et al., 2014).

En los últimos 10 años se ha documentado un incremento de $10 \%$ de los mamíferos de Oaxaca con un promedio de registro de 2 especies por año. En 2004 se reconocían 190 especies (Briones-Salas y Sánchez-Cordero, 2004) a las que se le adicionan Vampyrum spectrum (Alfaro, García-García y SantosMoreno, 2005), Peropteryx kappleri y Molossus molossus (Olguín, León-Paniagua, Samper-Palacios y Sánchez-Cordero, 2008), Conepatus semistriatus (Lira y Sánchez-Cordero, 2006), Eptesicus brasiliensis (García-García, Santos-Moreno, Alfaro y Soto-Centeno, 2007), Otospermophilus variegatus (Botello, Illoldi-Rangel, Linaje y Sánchez-Cordero, 2007), Centronycteris centralis y Natalus lanatus (Santos-Moreno, García-Orozco y Pérez-Cruz, 2010), Nyctinomops macrotis (Alfaro y Santos-Moreno, 2012), Molossus sinaloae y M. molossus (Briones-Salas, Peralta-Pérez y García-Luis, 2013), Phyllostomus stenops, Micronycteris schmidtorum y Myotis albescens (Santos-Moreno y Gallardo, 2014) y Eumops nanus (Torres-Morales, Rodríguez-Aguilar, Cabrera-Cruz y VillegasPatraca, 2014). 
Aunque en menor medida, en los últimos años las revisiones taxonómicas han tenido un efecto sobre el número de especies documentado en el estado. Actualmente se reconoce a Sorex ixtlanensis (Carraway, 2007), Megadontomys thomasi y M. nelsoni, estas últimas previamente habían sido identificadas como M. cryophilus (Vallejo y González-Cózatl, 2012). Revisiones de otros taxones sugieren la existencia de especies crípticas del género Habromys y de Peromyscus, particularmente en la Sierra Madre de Oaxaca (Ávila-Valle et al., 2012; León-Paniagua, Navarro-Sigüenza, Hernández-Baños y Morales, 2007).

En este trabajo se reconocen 96 especies de murciélagos, lo que difiere con las reconocidas por otros autores (GarcíaGrajales y Buenrostro, 2012), debido a que enlistan 5 especies que son sinónimos o su distribución se encuentra al norte de México (Mimon crenulatum/Mimon cozumelae, Artibeus intermedius = Artibeus lituratus, Rhogeessa aeneus/Rhogeessa tumida, Lasiurus borealis/Lasiurus blossevillii, Natalus stramineus $=$ Natalus mexicanus $)$. Asimismo, debido a las revisiones taxonómicas recientes y nuevos registros para el estado, las cifras registradas en el presente estudio contrastan con lo documentado por Sánchez-Cordero et al. (2014), donde mencionan 191 especies de mamíferos terrestres para Oaxaca, compuestas por 108 no voladores y 83 voladores.

Oaxaca es un área importante de endemismo de mamíferos (Escalante, 2003), la mayoría de las especies son del orden Rodentia, lo que es de esperarse, ya que tienen baja movilidad en comparación con otros órdenes (Ceballos y Oliva, 2005). Las subprovincias con una fisiografía montañosa (e.g., Sierra Madre de Oaxaca y la Sierra Madre del Sur) o que presentan una estacionalidad marcada (e.g., Planicie Costera del Pacífico, Planicie Costera del Istmo de Tehuantepec), son las que tienen mayor número de mamíferos endémicos y altos niveles de endemismo en otros grupos taxonómicos como plantas compuestas, mariposas, aves, anfibios y reptiles (GonzálezPérez et al., 2004; Suárez-Mota y Villaseñor, 2011) y son reconocidas como zonas de importancia biológica (Ceballos y Oliva, 2005; Escalante, 2003; García-Marmolejo, Escalante y Morrone, 2008). Estas áreas tienen en común historias biogeográficas complejas de formación y aislamiento geográfico y climático, factores que promueven la especiación (Halffter, Llorente-Bousquets y Morrone, 2008; Luna-Vega, 2008; Toledo, 1976). Sin embargo, dichas zonas se encuentran amenazadas por la demanda de recursos y por la fragmentación y transformación del hábitat, especialmente en el Istmo de Tehuantepec (Velázquez et al., 2003). Es importante que las estrategias de conservación en Oaxaca sean evaluadas para determinar si cumplen con la protección de especies restringidas o en alguna categoría de riesgo, así como la protección de la vegetación primaria, y que las nuevas propuestas relacionen la diversidad y distribución de estos mamíferos (Sosa-Escalante et al., 2014).

Además, algunas subprovincias de Oaxaca han sido exploradas más que otras y continuamente (e.g., Sierra Madre de Oaxaca, Montañas y Valles del Centro), por lo que es necesario poner énfasis en aquellos sitios con pocas localidades de recolecta, particularmente al oeste del estado, en la Depresión del Balsas y las Montañas y Valles del Occidente, ya que la falta de recolecta en algunas zonas del estado impide una evaluación objetiva acerca de la existencia de especies que puedan estar bajo alguna categoría de riesgo o sean endémicas del estado. Los resultados del análisis de similitud entre las subprovincias están en concordancia con regionalizaciones biogeografías de América (Morrone, 2001): el nodo formado por las subprovincias de la vertiente del Pacífico y las subprovincias del centro de Oaxaca se ubican en la provincia Costa Pacífica Mexicana y en la Sierra Madre del Sur, respectivamente; mientras que el otro nodo que corresponde a las subprovincias de la vertiente del Atlántico está en la provincia Golfo de México. Además, resultó notable que la subprovincia Fosa de Tehuacán se mantuviera independiente de los 2 principales nodos, por su posición dentro de la provincia Cuenca del Balsas.

La mayor similitud mastofaunística entre la Sierra Madre del Sur y Planicie Costera del Pacífico puede explicarse porque se extienden en paralelo a la costa y su contacto es continuo, aumentando el intercambio de especies. También se observó mayor similitud entre las que poseen climas similares como: Valles Centrales de Oaxaca y Montañas y Valles del Occidente; Sierra Madre del Sur de Oaxaca y Chiapas y Planicie Costera del Golfo. Por otro lado, la disimilitud entre el resto de las subprovincias indica que existe un alto recambio de especies, como ha sido observado en otros grupos y explica la alta diversidad beta en México y particularmente en Oaxaca (Koleff y Soberón, 2008; Rodríguez, Soberón y Arita, 2003).

\section{Agradecimientos}

Al Instituto Politécnico Nacional por financiar parcialmente el proyecto (SIP: 20100263, 20110547, 20121142). Al Consejo Nacional de Ciencia y Tecnología por las becas otorgadas a M.L.N. y a M.C.M. para realizar estudios de posgrado. M.B.S. agradece al Sistema de Becas de exclusividad y al programa de Estímulos al Desempeño a la Investigación del Instituto Politécnico Nacional por el apoyo. De igual forma, al Sistema Nacional de Investigadores por el reconocimiento. Asimismo, a J. SosaEscalante, quien realizó las observaciones y recomendaciones que ayudaron a mejorar el presente trabajo.

\section{Anexo.}

Lista taxonómica de los mamíferos terrestres de Oaxaca. Claves: subprovincia fisiográfica: DB: Depresión del Balsas; DIT: Depresión Ístmica de Tehuantepec; FT: Fosa de Tehuacán; MVC: Montañas y Valles del Centro; MVO: Montañas y Valles del Occidente; PCG: Planicie Costera del Golfo; PCP: Planicie Costera del Pacífico; PCT: Planicie Costera de Tehuantepec; SMO: Sierra Madre de Oaxaca; SMOC: Sierra Madre del Sur de Oaxaca y Chiapas; SMS: Sierra Madre del Sur; VC: Valles Centrales. Distrito: Centro (1), Choapan (2), Coixtlahuaca (3), Cuicatlán (4), Ejutla (5), Etla (6), Huajuapán (7), Ixtlán (8), Jamiltepec (9), Juchitán (10), Juquila (11), Juxtlahuaca (12), Miahuatlán (13), Mixe (14), Nochixtlán (15), Ocotlán (16), Pochutla (17), Putla (18), Silacayoapan (19), Sola de Vega (20), Tehuantepec (21), Teotitlán (22), Teposcolula (23), Tlacolula (24), Tlaxiaco (25), Tuxtepec (26), Villa Alta (27), Yautepec (28), Zaachila (29), Zimatlán (30). Vegetación: BC: Bosque 
de coníferas; BE: Bosque espinoso; BTC: Bosque tropical caducifolio; BMM: Bosque mesófilo de montaña; BTP: Bosque tropical perennifolio; BTS: Bosque tropical subcaducifolio; CAF: Cafetales; CUL: Cultivo; MX: Matorral xerófilo; PAS: Pastizal; VS: Vegetación secundaria. Altitud (metros): $1=0-500$, $2=501-1,000,3=1001-1,500,4=1501-2000,5=2001-2500$, $6=2,501-3,000,7=>3,500$. Estado de conservación: IUCN: CR: en peligro crítico; EN: en peligro; EW: extinta en vida silvestre; NT: casi amenazada; VU: vulnerable. CITES: I, II, III. NOM (NOM-059-SEMARNAT-2010): A: amenazada; E: probablemente extinta en medio silvestre; P: en peligro de extinción; PR: sujeta a protección especial. Endemismo: $\mathrm{MX}=$ endémico de México, OAX = endémico de Oaxaca. Se marca con asterisco $(*)$ las especies prioritarias para la conservación presentes en Oaxaca de acuerdo con la Semarnat (2014).

\begin{tabular}{|c|c|c|c|c|c|c|c|}
\hline Lista sistemática & Subprovincia fisiográfica & Distrito & Vegetación & Altitud & $\mathrm{NOM}$ & IUCN & Cites \\
\hline \multicolumn{8}{|l|}{ Orden Didelphimorphia } \\
\hline \multicolumn{8}{|l|}{ Familia Didelphidae } \\
\hline $\begin{array}{l}\text { Caluromys derbianus } \\
\text { (Waterhouse, 1841) }\end{array}$ & & & & & A & & \\
\hline $\begin{array}{l}\text { Caluromys derbianus aztecus } \\
\text { (Thomas, 1913) }\end{array}$ & PCG, SMOC & 10 & BTP,BTS & 1 & & & \\
\hline $\begin{array}{l}\text { Chironectes minimus } \\
\quad \text { (Zimmermann, 1780) }\end{array}$ & & & & & $\mathrm{P}$ & & \\
\hline $\begin{array}{l}\text { Chironectes minimus } \\
\quad \text { argyrodytes Dickey, } 1928\end{array}$ & SMO & 10 & BTP,BTS & 1 & & & \\
\hline \multicolumn{8}{|l|}{ Didelphis marsupialis } \\
\hline $\begin{array}{l}\text { Didelphis marsupialis caucae } \\
\text { J. A. Allen, } 1900\end{array}$ & $\begin{array}{l}\text { DIT,FT,MVC, } \\
\text { PCG,PCT, } \\
\text { PCP,SMO, } \\
\text { SMOC,SMS,VC }\end{array}$ & $\begin{array}{l}1,2,4,8,9 \\
10,11,17 \\
21,22,24 \\
26,27\end{array}$ & $\begin{array}{l}\text { BC,BTP,MX,CUL, } \\
\text { VS,BTC,BTS }\end{array}$ & $1,2,3,4,5,6$ & & & \\
\hline \multicolumn{8}{|l|}{$\begin{array}{l}\text { Didelphis virginiana Kerr, } \\
\quad 1792\end{array}$} \\
\hline $\begin{array}{l}\text { Didelphis virginiana } \\
\quad \text { californica } \text { Bennett, } 1833\end{array}$ & $\begin{array}{l}\text { DB,DIT,FT, } \\
\text { MVO,PCG, } \\
\text { PCT,PCP,SMO, } \\
\text { SMOC,SMS,VC }\end{array}$ & $\begin{array}{l}1,4,7,8,9 \\
10,11,17 \\
21,22,26,29\end{array}$ & $\begin{array}{l}\text { BC,CUL,MX, } \\
\text { BE,BTS, } \\
\text { BTC,CAF }\end{array}$ & $1,2,3,4,6$ & & & \\
\hline \multicolumn{8}{|l|}{$\begin{array}{r}\text { Philander opossum } \\
\text { (Linnaeus, 1758) }\end{array}$} \\
\hline $\begin{array}{l}\text { Philander opossum } \\
\text { fuscogriseus (J. A. Allen, } \\
\text { 1900) }\end{array}$ & $\begin{array}{l}\text { DIT,PCG,PCT, } \\
\text { PCP,SMO,SMOC }\end{array}$ & $8,10,11,21,26$ & BC,BMM,BTP,BTS & $1,2,3$ & & & \\
\hline \multicolumn{8}{|l|}{$\begin{array}{l}\text { Marmosa mexicana Merriam, } \\
1897\end{array}$} \\
\hline $\begin{array}{l}\text { Marmosa mexicana mexicana } \\
\text { Merriam, } 1897\end{array}$ & $\begin{array}{l}\text { PCT,PCP, } \\
\text { SMO,SMOC,SMS }\end{array}$ & $\begin{array}{l}1,8,10,11,13 \\
17,21,22,26,27\end{array}$ & $\begin{array}{l}\text { BC,CAF,BMM,VS, } \\
\text { BTS,CUL,BTP }\end{array}$ & $1,2,3,4,5,6,7$ & & & \\
\hline \multicolumn{8}{|l|}{$\begin{array}{l}\text { Tlacuatzin canescens }(\mathrm{J} . \text { A. } \\
\text { Allen, } 1893)^{\mathrm{MX}}\end{array}$} \\
\hline $\begin{array}{l}\text { Tlacuatzin canescens } \\
\text { canescens }(\mathrm{J} . \text { A. Allen, } \\
\text { 1893) }\end{array}$ & $\begin{array}{l}\text { DIT,MVC,MVO,PCT, } \\
\text { PCP,SMO,SMS, } \\
\text { VC }\end{array}$ & $\begin{array}{l}1,8,9,10,11 \\
17,19,21,26\end{array}$ & $\begin{array}{l}\text { BC,BMM,CUL, } \\
\text { BTS,BTC,MX,VS }\end{array}$ & $1,2,3,4$ & & & \\
\hline \multicolumn{7}{|l|}{$\begin{array}{l}\text { Dasypus novemcinctus } \\
\text { Linnaeus, } 1758\end{array}$} & \\
\hline $\begin{array}{l}\text { Dasypus novemcinctus } \\
\quad \text { mexicanus Peters, } 1864\end{array}$ & $\begin{array}{l}\text { DIT,MVO,PCG,PCT, } \\
\text { PCP,SMO,SMOC, } \\
\text { SMS,VC }\end{array}$ & $\begin{array}{l}2,4,6,7,9,10,11, \\
17,21,22 \\
26,27,28\end{array}$ & $\begin{array}{l}\text { BTS,BTC,CAF,VS, } \\
\text { MX,CUL,BE,DC }\end{array}$ & $1,2,3,4,5$ & & & \\
\hline $\begin{array}{l}\text { Orden Pilosa } \\
\text { Familia Myrmecophagidae } \\
\text { Tamandua mexicana (De } \\
\quad \text { Saussure, 1860) }\end{array}$ & & & & & & & \\
\hline $\begin{array}{l}\text { Tamandua mexicana } \\
\text { mexicana (De Saussure, } \\
\text { 1860) }\end{array}$ & $\begin{array}{l}\text { MVC,PCT,PCP, } \\
\text { SMO,SMOC,SMS }\end{array}$ & $\begin{array}{l}8,9,10,11,17 \\
21,24,26,27,28\end{array}$ & $\begin{array}{l}\text { BC,DC,CUL,BE, } \\
\text { BTS,BTC,CAF }\end{array}$ & $1,2,3,4$ & $\mathrm{P}$ & & \\
\hline
\end{tabular}




\begin{tabular}{|c|c|c|c|c|c|c|c|}
\hline Lista sistemática & Subprovincia fisiográfica & Distrito & Vegetación & Altitud & NOM & IUCN & Cites \\
\hline \multicolumn{8}{|l|}{ Familia Cyclopedidae } \\
\hline $\begin{array}{l}\text { Cyclopes didactylus } \\
\text { (Linnaeus, 1758) }\end{array}$ & & & & & $\mathrm{P}$ & & \\
\hline $\begin{array}{l}\text { Cyclopes didactylus } \\
\quad \text { mexicanus } \text { Hollister, } 1914\end{array}$ & PCG,PCT,PCP & 10,21 & BE,BTC,CUL,VS & 1 & & & \\
\hline \multicolumn{8}{|l|}{ Orden Soricomorpha } \\
\hline \multicolumn{8}{|l|}{ Familia Soricidae } \\
\hline $\begin{array}{l}\text { Cryptotis goldmani } \\
\quad(\text { Merriam, 1895) } \mathrm{MX}\end{array}$ & & & & & $\operatorname{Pr}$ & & \\
\hline $\begin{array}{l}\text { Cryptotis goldmani machetes } \\
\quad \text { (Merriam, 1895) }\end{array}$ & $\begin{array}{l}\text { MVO,PCT,PCP, } \\
\text { SMO,SMS }\end{array}$ & $\begin{array}{l}4,8,11,13 \\
14,17,18,21\end{array}$ & $\begin{array}{l}\text { BC,BMM,CUL, } \\
\text { BTP,BTS,BTC }\end{array}$ & $1,2,3,4,5,6,7$ & & & \\
\hline $\begin{array}{l}\text { Cryptotis magnus (Merriam, } \\
\text { 1895) OAX }\end{array}$ & $\begin{array}{l}\text { DIT,MVC,PCG, } \\
\text { SMO }\end{array}$ & $4,6,8,10,26$ & $\mathrm{BC}, \mathrm{BMM}$ & $1,2,3,4,5,6$ & $\operatorname{Pr}$ & $\mathrm{VU}$ & \\
\hline $\begin{array}{l}\text { Cryptotis mexicanus (Coues, } \\
\text { 1877) } \mathrm{MX}\end{array}$ & $\begin{array}{l}\text { DIT,FT,MVO,PCP, } \\
\text { SMO,SMOC,SMS,VC }\end{array}$ & $\begin{array}{l}1,4,8,10,11,13 \\
14,17,20,22,25,27\end{array}$ & BC,BMM,CUL,MX & $1,2,3,4,5,6,7$ & & & \\
\hline \multicolumn{8}{|l|}{ Cryptotis parvus (Say, 1822) } \\
\hline $\begin{array}{l}\text { Cryptotis parvus pueblensis } \\
\text { Jackson, } 1933\end{array}$ & $\begin{array}{l}\text { FT,MVC,MVO,PCT, } \\
\text { PCP,SMO,SMS }\end{array}$ & $\begin{array}{l}8,11,13,14,17 \\
21,22,25,27,30\end{array}$ & $\begin{array}{l}\text { BC,BMM,CUL, } \\
\text { MX,BTC,BTS }\end{array}$ & $1,2,3,4,5,6$ & & & \\
\hline $\begin{array}{l}\text { Cryptotis peregrina } \\
\quad \text { (Merriam, 1895) } \mathrm{OAX}\end{array}$ & $\mathrm{MVO}, \mathrm{VC}$ & $1,20,25,29,30$ & $\mathrm{BC}, \mathrm{BMM}$ & $4,5,6$ & $\operatorname{Pr}$ & & \\
\hline $\begin{array}{l}\text { Cryptotis phillipsii } \\
\quad \text { (Schaldach, 1966) OAX }\end{array}$ & PCP,SMS,VC & $11,13,17$ & $\mathrm{BC}$ & $1,2,3,4,5$ & & & \\
\hline $\begin{array}{l}\text { Megasorex gigas (Merriam, } \\
\text { 1897) MX }\end{array}$ & MVO & 18 & CUL & 3 & A & & \\
\hline $\begin{array}{l}\text { Sorex ixtlanensis Carraway, } \\
2007 \text { MX }\end{array}$ & MVO,SMO,VC & $8,15,30$ & $\mathrm{BC}$ & 5 & & & \\
\hline $\begin{array}{l}\text { Sorex ventralis Merriam, } 189 \\
\text { MX }\end{array}$ & SMO,SMS,VC & $1,8,13,27,29$ & BC,CUL & $3,4,5,6,8$ & & & \\
\hline $\begin{array}{l}\text { Sorex salvini Merriam, } 187 \\
\quad \text { MX }\end{array}$ & $\begin{array}{l}\text { MVO,PCP,PCT, } \\
\text { SMO,SMS,VC }\end{array}$ & $1,9,13,22,30$ & $\mathrm{BC}$ & $1,3,4,5,6$ & & & \\
\hline $\begin{array}{l}\text { Sorex salvini altoensis } \\
\quad \text { Carraway, } 2007\end{array}$ & MVO & 19,23 & $\mathrm{BC}$ & 3,5 & & & \\
\hline $\begin{array}{l}\text { Sorex salvini oaxacae } \\
\text { Jackson, } 1925 \text { OAX }\end{array}$ & MVO,SMO,SMS & $8,13,15,17$ & $\mathrm{BC}$ & $3,4,5,6$ & A & & \\
\hline $\begin{array}{l}\text { Sorex salvini veraecrucis } \\
\quad \text { Jackson, } 1925\end{array}$ & PCT,SMO,VC & $1,8,14,21,22,27$ & $\mathrm{BC}$ & $1,3,4,5,6$ & PR & & \\
\hline $\begin{array}{l}\text { Sorex veraepacis Alston, } \\
\quad 1877\end{array}$ & & & $\mathrm{BC}$ & & & & \\
\hline $\begin{array}{l}\text { Sorex veraepacis mutabilis } \\
\quad \text { Meriam, } 189 \mathrm{MX}\end{array}$ & $\begin{array}{l}\text { PCT,PCP,SMO, } \\
\text { SMS,VC }\end{array}$ & $\begin{array}{l}1,4,8,11,13 \\
14,21,27\end{array}$ & $\begin{array}{l}\text { BC,BMM,BTP, } \\
\text { BTS,CUL }\end{array}$ & $1,3,4,5,6,7$ & A & & \\
\hline \multicolumn{8}{|l|}{ Orden Chiroptera } \\
\hline $\begin{array}{l}\text { Balantiopteryx io Thomas, } \\
1904\end{array}$ & DIT,PCG,SMOC & $2,10,26$ & BTP,BTS,CUL & 1 & & VU & \\
\hline \multicolumn{8}{|l|}{$\begin{array}{l}\text { Balantiopteryx plicata Peters, } \\
1867\end{array}$} \\
\hline $\begin{array}{l}\text { Balantiopteryx plicata plicata } \\
\text { Peters, } 1867\end{array}$ & $\begin{array}{l}\text { DIT,FT,MVC,PCG, } \\
\text { PCT,PCP,SMO, } \\
\text { SMOC,SMS,VC }\end{array}$ & $\begin{array}{l}1,2,4,8,10,11,14, \\
17,18,21,22,24, \\
26,27,28\end{array}$ & $\begin{array}{l}\text { BTS,BTC,VS,DC, } \\
\text { BE,MX,BTP,CUL }\end{array}$ & $1,2,3,4,6$ & & & \\
\hline $\begin{array}{l}\text { Centronycteris centralis } \\
\text { Thomas, } 1912\end{array}$ & DIT,PCT & 10 & BTC,CUL & 1 & $\operatorname{Pr}$ & & \\
\hline \multicolumn{8}{|l|}{ Diclidurus albus } \\
\hline $\begin{array}{l}\text { Diclidurus albus virgo } \\
\quad \text { Thomas, } 1903\end{array}$ & PCG & & BTP & & & & \\
\hline \multicolumn{8}{|l|}{$\begin{array}{l}\text { Peropteryx kappleri Peters, } \\
1867\end{array}$} \\
\hline $\begin{array}{l}\text { Peropteryx kappleri kappleri } \\
\text { Peters, } 1867\end{array}$ & DIT,SMOC & 10 & BTP & 1 & $\operatorname{Pr}$ & & \\
\hline $\begin{array}{l}\text { Peropteryx macrotis (J. A. } \\
\text { Wagner, 1843) }\end{array}$ & PCG,PCT,SMOC & $10,14,21,26$ & BTP,BTS & 1 & & & \\
\hline
\end{tabular}




\begin{tabular}{|c|c|c|c|c|c|c|c|}
\hline Lista sistemática & Subprovincia fisiográfica & Distrito & Vegetación & Altitud & NOM & IUCN & Cites \\
\hline $\begin{array}{l}\text { Rhynchonycteris naso } \\
\text { (Wied-Neuwied, 1820) }\end{array}$ & DIT,SMOC & 10 & BTP,BTS & 1 & $\operatorname{Pr}$ & & \\
\hline $\begin{array}{l}\text { Saccopteryx bilineata } \\
\quad \text { (Temminck, 1838) }\end{array}$ & $\begin{array}{l}\text { DIT,PCG,PCT, } \\
\text { PCP,SMO,SMOC }\end{array}$ & $\begin{array}{l}9,10,11,14 \\
17,21,22,26\end{array}$ & $\begin{array}{l}\text { CUL,DC, } \\
\text { BTCBE,BTP }\end{array}$ & $1,2,3$ & & & \\
\hline \multicolumn{8}{|l|}{ Familia Molossidae } \\
\hline $\begin{array}{l}\text { Cynomops mexicanus (J. K. } \\
\text { Jones y Genoways, 1967) } \\
\text { MX }\end{array}$ & PCT,SMS & 10,13 & $\mathrm{BC}$ & 1,5 & $\operatorname{Pr}$ & & \\
\hline \multicolumn{8}{|l|}{$\begin{array}{l}\text { Eumops auripendulus (Shaw, } \\
\text { 1800) }\end{array}$} \\
\hline $\begin{array}{l}\text { Eumops auripendulus } \\
\quad \text { auripendulus (Shaw, 1800) }\end{array}$ & SMO & 14 & CUL & 2 & & & \\
\hline Eumops nanus (Miller, 1900) & PCT & 10 & CUL, BTC & 1 & $\operatorname{Pr}$ & & \\
\hline \multicolumn{8}{|l|}{$\begin{array}{l}\text { Eumops underwoodi } \\
\text { Goodwin, } 1940\end{array}$} \\
\hline $\begin{array}{l}\text { Eumops underwoodi } \\
\text { underwoodi Goodwin, } \\
1940\end{array}$ & MVC,PCP,SMS & $13,21,28$ & $\mathrm{BC}, \mathrm{BTC}, \mathrm{BE}$ & $1,2,3$ & & & \\
\hline $\begin{array}{l}\text { Molossus aztecus De } \\
\text { Saussure, } 1860\end{array}$ & $\begin{array}{l}\text { MVC,PCG,PCT, } \\
\text { PCP,SMOC,SMS }\end{array}$ & $\begin{array}{l}9,10,13,17 \\
21,26,28\end{array}$ & $\begin{array}{l}\text { BC,BTP,BTC, } \\
\text { BE,BTS }\end{array}$ & $1,2,4$ & & & \\
\hline $\begin{array}{l}\text { Molossus molossus (Pallas, } \\
\text { 1766) }\end{array}$ & PCT & 10 & BTC,CUL & 1 & & & \\
\hline \multicolumn{8}{|l|}{$\begin{array}{l}\text { Molossus rufus E. Geoffroy } \\
\text { Saint-Hilaire, } 1805\end{array}$} \\
\hline $\begin{array}{l}\text { Molossus rufus nigricans } \\
\quad \text { Miller, } 1902\end{array}$ & $\begin{array}{l}\text { DIT,MVC,PCG,PCT, } \\
\text { PCP,SMO,SMOC, SMS }\end{array}$ & $\begin{array}{l}8,10,14,17 \\
21,26,28\end{array}$ & $\begin{array}{l}\text { BTP,CUL,DC, } \\
\text { BTC,BE,VS }\end{array}$ & $1,2,5$ & & & \\
\hline \multicolumn{8}{|l|}{$\begin{array}{l}\text { Molossus sinaloae J. A. } \\
\text { Allen, } 1906\end{array}$} \\
\hline $\begin{array}{l}\text { Molossus sinaloae sinaloae J. } \\
\text { A. Allen, } 1906\end{array}$ & PCT & 10 & BTC,CUL & 1 & & & \\
\hline $\begin{array}{l}\text { Nyctinomops aurispinosus } \\
\quad \text { (Peale, 1848) }\end{array}$ & FT,PCT & 4,10 & BTC,BTS,MX & 1,2 & & & \\
\hline $\begin{array}{l}\text { Nyctinomops laticaudatus (E. } \\
\text { Geoffroy Saint-Hilaire, } \\
\text { 1805) }\end{array}$ & MVC,PCT,SMS & 10,21 & & 1 & & & \\
\hline $\begin{array}{l}\text { Nyctinomops laticaudatus } \\
\text { ferrugineus (Goodwin, } \\
\text { 1954) }\end{array}$ & $\begin{array}{l}\text { DIT,MVC,PCT, } \\
\text { SMS }\end{array}$ & 10,21 & BTC,BTS,CUL & 1 & & & \\
\hline $\begin{array}{l}\text { Nyctinomops macrotis (Gray, } \\
\text { 1840) }\end{array}$ & $\mathrm{VC}$ & 24 & BTC & 4 & & & \\
\hline \multicolumn{8}{|l|}{$\begin{array}{l}\text { Promops centralis Thomas, } \\
1915\end{array}$} \\
\hline $\begin{array}{l}\text { Promops centralis centralis } \\
\text { Thomas, } 1915\end{array}$ & VC, PCP, PCT, PCG & $1,6,21,24,26$ & $\begin{array}{l}\text { CUL,BTC, } \\
\text { BE,VS }\end{array}$ & $1,4,5$ & & & \\
\hline $\begin{array}{l}\text { Tadarida brasiliensis (I. } \\
\text { Geoffroy Saint-Hilaire, } \\
\text { 1824) }\end{array}$ & $\begin{array}{l}\text { DIT,MVC,MVO,PCT, } \\
\text { SMO,SMOC,SMS,VC }\end{array}$ & $\begin{array}{l}1,8,10,13,20 \\
21,24,29\end{array}$ & & $1,2,4,5,6$ & & & \\
\hline $\begin{array}{l}\text { Tadarida brasiliensis } \\
\text { mexicana (De Saussure, } \\
\text { 1860) }\end{array}$ & $\begin{array}{l}\text { FT,MVC,MVO, } \\
\text { PCP,SMO,VC }\end{array}$ & $\begin{array}{l}1,6,8,15,21 \\
22,25,27\end{array}$ & & $1,3,4,5,6,7$ & & & \\
\hline \multicolumn{8}{|l|}{ Familia Natalidae } \\
\hline $\begin{array}{l}\text { Natalus lanatus Tejedor, } \\
2005^{\mathrm{MX}}\end{array}$ & SMOC & 10 & BTP & 1 & & & \\
\hline $\begin{array}{l}\text { Natalus mexicanus Miller, } \\
\quad 1902\end{array}$ & $\begin{array}{l}\text { DIT,MVC,MVO,PCG, } \\
\text { PCT,PCP,SMO, } \\
\text { SMOC,SMS }\end{array}$ & $10,11,20,21$ & $\begin{array}{l}\text { BTC,BE,BTS, } \\
\text { CUL,VS }\end{array}$ & $1,2,3,4$ & & & \\
\hline \multicolumn{8}{|l|}{$\begin{array}{l}\text { Familia Mormoopidae } \\
\text { Mormoops megalophylla } \\
\text { (Peters, 1864) }\end{array}$} \\
\hline $\begin{array}{l}\text { Mormoops megalophylla } \\
\text { megalophylla (Peters, } \\
\text { 1864) }\end{array}$ & $\begin{array}{l}\text { FT,MVC,MVO,PCT, } \\
\text { PCP,SMO,SMS }\end{array}$ & $4,7,10,21$ & $\begin{array}{l}\text { BTC,BTP,CUL, } \\
\text { MX,DC,BC,BTC, } \\
\text { BE,BTS }\end{array}$ & $1,2,3,4,5$ & & & \\
\hline
\end{tabular}




\begin{tabular}{|c|c|c|c|c|c|c|c|}
\hline Lista sistemática & Subprovincia fisiográfica & Distrito & Vegetación & Altitud & $\mathrm{NOM}$ & IUCN & Cites \\
\hline \multicolumn{8}{|l|}{ Pteronotus davyi Gray, 1838} \\
\hline $\begin{array}{l}\text { Pteronotus davyi } \\
\quad \text { fulvus(Thomas, 1892) }\end{array}$ & $\begin{array}{l}\text { FT,MVC,MVO,PCG, } \\
\text { PCT,PCP,SMO, } \\
\text { SMOC,SMS }\end{array}$ & $\begin{array}{l}4,10,17,20 \\
21,24,26,30\end{array}$ & $\begin{array}{l}\text { BC,CUL,BTC, } \\
\text { BE,DC,MX,BTS,VS }\end{array}$ & $1,2,3,6$ & & & \\
\hline $\begin{array}{l}\text { Pteronotus parnellii (Gray, } \\
\text { 1843) }\end{array}$ & $\begin{array}{l}\text { DIT,FT,MVC,MVO, } \\
\text { PCG,PCT,PCP, } \\
\text { SMO,SMOC,SMS }\end{array}$ & $\begin{array}{l}4,7,8,9,10,11,13, \\
17,18,20,21,22,26,28\end{array}$ & $\begin{array}{l}\text { BTC,BTP,BTS, } \\
\text { DC,MX,CUL }\end{array}$ & $1,2,3,4,5,6$ & & & \\
\hline $\begin{array}{l}\text { Pteronotus parnellii } \\
\text { mesoamericanus Smith, } \\
1972\end{array}$ & PCG & 10 & BTP & 1 & & & \\
\hline $\begin{array}{l}\text { Pteronotus parnellii } \\
\quad \text { mexicanus (Miller, 1902) }\end{array}$ & $\begin{array}{l}\text { DIT,FT,MVC,MVO, } \\
\text { PCG,PCT,PCP, } \\
\text { SMO,SMOC,SMS }\end{array}$ & $\begin{array}{l}4,9,10,11,17,18 \\
20,21,22,26,28\end{array}$ & $\begin{array}{l}\text { BC,BTS,BTC, } \\
\text { DC,MX,CUL }\end{array}$ & $1,2,3,4,5$ & & & \\
\hline $\begin{array}{l}\text { Pteronotus personatus (J. A. } \\
\text { Wagner, 1843) }\end{array}$ & & & CUL & & & & \\
\hline $\begin{array}{l}\text { Pteronotus personatus } \\
\quad \text { psilotis (Dobson, 1878) }\end{array}$ & $\begin{array}{l}\text { PCG,PCT,PCP, } \\
\text { SMO,SMS }\end{array}$ & $10,11,21,26$ & BTC,BE,DC,VS & 1,2 & & & \\
\hline \multicolumn{8}{|l|}{ Familia Noctilionidae } \\
\hline \multicolumn{8}{|l|}{$\begin{array}{l}\text { Noctilio leporinus (Linnaeus, } \\
1758 \text { ) }\end{array}$} \\
\hline $\begin{array}{l}\text { Noctilio leporinus mastivus } \\
\text { (Vahl, 1797) }\end{array}$ & $\begin{array}{l}\text { MVC,PCT,PCP, } \\
\text { SMO }\end{array}$ & $10,11,17,21$ & BTC,BE,DC,PAS,VS & 1,2 & & & \\
\hline \multicolumn{8}{|l|}{ Familia Phyllostomidae } \\
\hline \multicolumn{8}{|l|}{$\begin{array}{l}\text { Carollia perspicillata } \\
\text { (Linnaeus, 1758) }\end{array}$} \\
\hline $\begin{array}{l}\text { Carollia perspicillata azteca } \\
\text { De Saussure, } 1860\end{array}$ & $\begin{array}{l}\text { DIT,PCG,PCT, } \\
\text { SMO,SMOC,SMS }\end{array}$ & $\begin{array}{l}8,10,17 \\
21,22,26\end{array}$ & $\begin{array}{l}\text { BTP,BTS, } \\
\text { CAF,CUL,VS }\end{array}$ & $1,2,3,4$ & & & \\
\hline $\begin{array}{l}\text { Carollia sowelli R. J. Baker, } \\
\text { Solary y Hoffmann, } 2002\end{array}$ & $\begin{array}{l}\text { DIT,MVC,MVO, } \\
\text { PCG,PCT,PCP, } \\
\text { SMO,SMOC,SMS }\end{array}$ & $\begin{array}{l}2,8,10,14,17 \\
21,22,26,27,30\end{array}$ & $\begin{array}{l}\text { BMM,BTS,CAF, } \\
\text { BTP,CUL }\end{array}$ & $1,2,3,4,6$ & & & \\
\hline $\begin{array}{l}\text { Carollia subrufa (Hahn, } \\
\text { 1905) }\end{array}$ & $\begin{array}{l}\text { DIT,MVC,MVO, } \\
\text { PCG,PCT,PCP, } \\
\text { SMO,SMOC,SMS,VC }\end{array}$ & $\begin{array}{l}8,9,10,11 \\
13,17,18,21 \\
26,27,28,30\end{array}$ & $\begin{array}{l}\text { BTS,BTC,BTP, } \\
\text { CAF,VS,CUL,BE }\end{array}$ & $1,2,3,4,5,6$ & & & \\
\hline \multicolumn{8}{|l|}{$\begin{array}{l}\text { Desmodus rotundus }(\mathrm{E} . \\
\text { Geoffroy Saint-Hilaire, } \\
1810)\end{array}$} \\
\hline $\begin{array}{l}\text { Desmodus rotundus murinus } \\
\text { (E. Geoffroy Saint-Hilaire, } \\
\text { 1810) }\end{array}$ & $\begin{array}{l}\text { DIT,FT,MVC,MVO, } \\
\text { vPCG,PCT,PCP, } \\
\text { SMO,SMOC,SMS,VC }\end{array}$ & $\begin{array}{l}1,2,4,7,8,9,10,11, \\
13,14,17,18,20,21, \\
22,23,24,25,26,28,30\end{array}$ & $\begin{array}{l}\text { CUL,MX,BTP, } \\
\text { BTS,BTC,VS,DC,BC }\end{array}$ & $1,2,3,4,5,6$ & & & \\
\hline $\begin{array}{l}\text { Diphylla ecaudata Spix, } 1823 \\
\text { Anoura geoffroyi Gray, } 1838\end{array}$ & PCG,PCT,PCP & 10,21 & $\begin{array}{l}\text { BTC,CUL,BE,DC } \\
\text { BC,BTS,CAF }\end{array}$ & 1 & & & \\
\hline $\begin{array}{l}\text { Anoura geoffroyi lasiopyga } \\
\quad \text { (Peters, 1868) }\end{array}$ & $\begin{array}{l}\text { FT,MVO,PCG,PCP, } \\
\text { SMO,SMOC, } \\
\text { SMS,VC }\end{array}$ & $\begin{array}{l}1,4,6,7,8,10,11 \\
14,15,16,17,18,20 \\
23,25,26,30\end{array}$ & BTC,BC,MX,CUL & $1,2,3,4,5,6,7$ & & & \\
\hline $\begin{array}{l}\text { Choeroniscus godmani } \\
\text { (Thomas, 1903) }\end{array}$ & $\begin{array}{l}\text { DIT,MVO,PCT, } \\
\text { SMO,SMOC }\end{array}$ & $7,8,10,20$ & BC,BTP,BTS & $1,3,4,5$ & & & \\
\hline $\begin{array}{l}\text { Choeronycteris mexicana } \\
\text { Tschudi, } 1844\end{array}$ & $\begin{array}{l}\text { FT,MVC,MVO,PCG, } \\
\text { PCT,SMO,SMS,VC }\end{array}$ & $\begin{array}{l}1,4,6,7,11,15 \\
16,21,23,24,26\end{array}$ & BC,MX,CUL & $1,2,4,5,7$ & A & NT & \\
\hline $\begin{array}{l}\text { Hylonycteris underwoodi } \\
\text { Thomas, } 1903\end{array}$ & $\begin{array}{l}\text { MVC,MVO,PCG, } \\
\text { PCP,SMO,SMOC, } \\
\text { SMS }\end{array}$ & $10,11,17,20,26$ & $\begin{array}{l}\text { BC,BMM,BTP, } \\
\text { BTS,BTC,CUL }\end{array}$ & $1,2,3,4,5$ & & & \\
\hline $\begin{array}{l}\text { Hylonycteris underwoodi } \\
\text { minor Phillips y Jones, } \\
1971\end{array}$ & $\begin{array}{l}\text { MVC,PCP,SMO, } \\
\text { SMS }\end{array}$ & $8,11,17,28$ & $\begin{array}{l}\text { BC,BMM,BTP, } \\
\text { BTS,BTC,CUL }\end{array}$ & $1,2,4,5$ & & & \\
\hline $\begin{array}{l}\text { Hylonycteris underwoodi } \\
\text { underwoodi Thomas, } 1903\end{array}$ & PCG,SMO,SMOC & $8,10,26$ & BC,BTP & 1,3 & & & \\
\hline $\begin{array}{l}\text { Glossophaga commissarisi } \\
\text { Gardner, } 1962\end{array}$ & & & & & & & \\
\hline $\begin{array}{l}\text { Glossophaga commissarisi } \\
\text { commissarisi Gardner, } \\
1962\end{array}$ & $\begin{array}{l}\text { DIT,MVC,PCG,PCT, } \\
\text { PCP,SMO,SMOC,SMS }\end{array}$ & $\begin{array}{l}8,9,10,11 \\
18,21,26,27\end{array}$ & DC,BTP,CUL & $1,2,3,6$ & & & \\
\hline
\end{tabular}




\begin{tabular}{|c|c|c|c|c|c|c|c|}
\hline Lista sistemática & Subprovincia fisiográfica & Distrito & Vegetación & Altitud & $\mathrm{NOM}$ & IUCN & Cites \\
\hline $\begin{array}{l}\text { Glossophaga leachii (Gray, } \\
\text { 1844) }\end{array}$ & $\begin{array}{l}\text { DIT,FT,MVC,MVO, } \\
\text { PCG,PCT,PCP, } \\
\text { SMO,SMOC,SMS,VC }\end{array}$ & $\begin{array}{l}4,8,9,10,11,13,18 \\
20,21,23,24,26,28,30\end{array}$ & $\begin{array}{l}\text { BTC,MX,BTS, } \\
\text { CUL,BTP,VS }\end{array}$ & $1,2,3,4,5,6$ & & & \\
\hline $\begin{array}{l}\text { Glossophaga morenoi } \\
\quad \text { Martínez y Villa, } 193 \text { MX }\end{array}$ & $\begin{array}{l}\text { DIT,MVC,MVO,PCT, } \\
\text { PCP,SMO, } \\
\text { SMOC,SMS,VC }\end{array}$ & $\begin{array}{l}7,8,10,11,13 \\
17,18,21,22,28\end{array}$ & DC,VS & $1,2,3,4,5,6$ & & & \\
\hline $\begin{array}{l}\text { Glossophaga morenoi } \\
\text { mexicana Webster y Jones, } \\
1980\end{array}$ & $\begin{array}{l}\text { DIT,MVC,PCT, } \\
\text { PCP,SMO, } \\
\text { SMOC,SMS }\end{array}$ & $8,10,17,18,21,28$ & $\begin{array}{l}\text { BTC,BTS,VS, } \\
\text { DC,BTP }\end{array}$ & $1,2,3$ & & & \\
\hline $\begin{array}{l}\text { Glossophaga morenoi } \\
\text { morenoi Martínez y Villa, } \\
1938\end{array}$ & MVO & 7 & & 4 & & & \\
\hline \multicolumn{8}{|l|}{$\begin{array}{l}\text { Glossophaga soricina (Pallas, } \\
\text { 1766) }\end{array}$} \\
\hline $\begin{array}{l}\text { Glossophaga soricina } \\
\text { handleyi Webster y Jones, } \\
1980\end{array}$ & $\begin{array}{l}\text { DIT,FT,MVC, } \\
\text { MVO,PCG, } \\
\text { PCT,PCP,SMO, } \\
\text { SMOC,SMS,VC }\end{array}$ & $\begin{array}{l}1,2,4,6,8,9,10,11 \\
16,17,18,21 \\
24,26,27,28,30\end{array}$ & $\begin{array}{l}\text { BC,BMM,BTS,BTC, } \\
\text { BE,CAF,MX, } \\
\text { CUL,BTP,VS,DC }\end{array}$ & $1,2,3,4,5,6$ & & & \\
\hline $\begin{array}{l}\text { Leptonycteris nivalis* (De } \\
\quad \text { Saussure, } 1860)\end{array}$ & $\begin{array}{l}\text { FT,MVC,PCT, } \\
\text { PCP,SMO,VC }\end{array}$ & $1,4,8,9,21,22$ & BTC,BC,MX & $1,2,4,5,6$ & A & EN & \\
\hline $\begin{array}{l}\text { Leptonycteris yerbabuenae* } \\
\text { Martínez y Villa, } 1940\end{array}$ & $\begin{array}{l}\text { DIT,FT,MVC,MVO, } \\
\text { PCG,PCT,PCP, } \\
\text { SMO,SMS,VC }\end{array}$ & $\begin{array}{l}1,4,6,7,8,9 \\
10,11,16,17,20,21 \\
24,26,30\end{array}$ & $\begin{array}{l}\text { BC,BTC,BTP, } \\
\text { BTC,BTS,CUL,MX }\end{array}$ & $1,2,3,4,5,6$ & A & VU & \\
\hline $\begin{array}{l}\text { Glyphonycteris sylvestris } \\
\text { Thomas, } 1896\end{array}$ & PCT & 10 & BTP & 1 & & & \\
\hline $\begin{array}{l}\text { Lonchorhina aurita Tomes, } \\
\qquad 1863\end{array}$ & & & & & A & & \\
\hline $\begin{array}{l}\text { Lonchorhina aurita aurita } \\
\text { Tomes, } 1863\end{array}$ & PCG,SMOC & 10,26 & BTP,CUL & 1 & & & \\
\hline \multicolumn{8}{|l|}{$\begin{array}{l}\text { Macrotus waterhousii Gray, } \\
1843\end{array}$} \\
\hline $\begin{array}{l}\text { Macrotus waterhousii } \\
\text { mexicanus } \text { De } \\
\text { Saussure, } 1860\end{array}$ & $\begin{array}{l}\text { FT,MVC,MVO,PCT, } \\
\text { PCP,SMO,SMS }\end{array}$ & $4,7,10,21$ & $\begin{array}{l}\text { BTC,DC,CUL, } \\
\text { MX,BTS,VS }\end{array}$ & $1,2,3,4,5$ & & & \\
\hline $\begin{array}{l}\text { Lampronycteris brachyotis } \\
\quad \text { (Dobson, 1879) }\end{array}$ & DIT & 10 & ВTP,BTS & 1 & A & & \\
\hline \multicolumn{8}{|l|}{$\begin{array}{l}\text { Micronycteris microtis Miller, } \\
1898\end{array}$} \\
\hline $\begin{array}{l}\text { Micronycteris microtis } \\
\quad \text { mexicana } \text { Miller, } 1898\end{array}$ & $\begin{array}{l}\text { DIT,FT,MVC,MVO, } \\
\text { PCG,PCT,PCP, } \\
\text { SMO,SMS }\end{array}$ & $\begin{array}{l}4,7,9,10,11 \\
13,14,17,18,21 \\
22,24,26,28\end{array}$ & $\begin{array}{l}\text { BTC,BTS,BTP, } \\
\text { MX,CUL,VS }\end{array}$ & $1,2,3,4$ & & & \\
\hline $\begin{array}{l}\text { Micronycteris schmidtorum } \\
\quad \text { Sanborn, } 1935\end{array}$ & SMOC & 10 & BTP & 1 & A & & \\
\hline $\begin{array}{l}\text { Trachops cirrhosus (Spix, } \\
\text { 1823) }\end{array}$ & & & & & A & & \\
\hline $\begin{array}{l}\text { Trachops cirrhosus coffini } \\
\text { Goldman, } 1925\end{array}$ & $\begin{array}{l}\text { DIT,PCG,PCT, } \\
\text { SMO,SMOC }\end{array}$ & $8,10,21,26$ & BTP,BTS,CUL & $1,2,3$ & & & \\
\hline $\begin{array}{l}\text { Lophostoma brasiliense } \\
\text { Peters, } 1867\end{array}$ & SMO,SMOC & 8,10 & BTP & 1,3 & A & & \\
\hline $\begin{array}{l}\text { Mimon cozumelae Goldman, } \\
1914\end{array}$ & PCG,SMO & $2,10,21,26$ & ВTP,BTS & 1,2 & A & & \\
\hline \multicolumn{8}{|l|}{$\begin{array}{l}\text { Phylloderma stenops Peters, } \\
\quad 1865\end{array}$} \\
\hline $\begin{array}{l}\text { Phylloderma stenops } \\
\text { septentrionalis Goodwin, } \\
1940\end{array}$ & SMOC & 10 & BTP & 1 & A & & \\
\hline $\begin{array}{l}\text { Phyllostomus discolor (J. A. } \\
\text { Wagner, 1843) }\end{array}$ & & & & & & & \\
\hline Phyllostomus discolor & DIT,PCG,PCT,SMO & $8,10,21,26$ & BTS,CUL & $1,2,3$ & & & \\
\hline
\end{tabular}




\begin{tabular}{|c|c|c|c|c|c|c|c|}
\hline Lista sistemática & Subprovincia fisiográfica & Distrito & Vegetación & Altitud & NOM & IUCN & Cites \\
\hline \multicolumn{8}{|l|}{$\begin{array}{l}\text { Chrotopterus auritus (Peters, } \\
1856 \text { ) }\end{array}$} \\
\hline $\begin{array}{l}\text { Chrotopterus auritus auritus } \\
\text { (Peters, 1856) }\end{array}$ & PCG,SMO & 26 & BTP,BTS & 1,2 & A & & \\
\hline $\begin{array}{l}\text { Vampyrum spectrum } \\
\text { (Linnaeus, 1758) }\end{array}$ & PCG,SMOC & 2,10 & BTP & 1 & $\mathrm{P}$ & NT & \\
\hline $\begin{array}{l}\text { Artibeus jamaicensis Leach, } \\
\quad 1821\end{array}$ & $\begin{array}{l}\text { DIT,FT,MVO,PCG, } \\
\text { PCT,PCP,SMO, } \\
\text { SMOC,SMS,VC }\end{array}$ & $\begin{array}{l}1,2,4,7,8,9 \\
10,11,17,18,21 \\
22,26,27,28,30\end{array}$ & $\begin{array}{l}\text { BC,BE,BTC, } \\
\text { BTP,BTS,CAF, } \\
\text { CUL,DC,MX,VS }\end{array}$ & $1,2,3,4,5,6$ & & & \\
\hline $\begin{array}{l}\text { Artibeus jamaicensis paulus } \\
\text { W. B. Davis, } 1970\end{array}$ & DIT,PCT,SMOC & 10 & ВTP & 1,2 & & & \\
\hline $\begin{array}{l}\text { Artibeus jamaicensis } \\
\quad \text { triomylus Handley, } 1966\end{array}$ & $\begin{array}{l}\text { MVO,PCG,PCT, } \\
\text { PCP,SMO,SMS }\end{array}$ & $\begin{array}{l}8,9,11,17,18 \\
20,21,26,28,30\end{array}$ & $\begin{array}{l}\text { BC,BTS,BTC, } \\
\text { BTP,BE,CUL, } \\
\text { CAF,VS,DC }\end{array}$ & $1,2,3,6$ & & & \\
\hline $\begin{array}{l}\text { Artibeus jamaicensis } \\
\text { yucatanicus J. A. Allen, } \\
1904\end{array}$ & $\begin{array}{l}\text { FT,MVO,PCG,PCT, } \\
\text { SMO,SMOC,SMS,VC }\end{array}$ & $\begin{array}{l}1,4,7,8,10 \\
21,22,26\end{array}$ & $\begin{array}{l}\text { BC,BTC,MX, } \\
\text { CUL,BTP,BTS }\end{array}$ & $1,2,3,4,5$ & & & \\
\hline \multicolumn{8}{|l|}{$\begin{array}{l}\text { Artibeus lituratus (Olfers, } \\
\text { 1818) }\end{array}$} \\
\hline $\begin{array}{l}\text { Artibeus lituratus palmarum } \\
\text { J. A. Allen y Chapman, } \\
1897\end{array}$ & $\begin{array}{l}\text { DIT,FT,MVC,MVO, } \\
\text { PCG,PCT,PCP, } \\
\text { SMO,SMOC,SMS,VC }\end{array}$ & $\begin{array}{l}1,2,4,6,7,8,9,10 \\
11,14,17,18,20,21 \\
22,26,27,28,30\end{array}$ & $\begin{array}{l}\text { BEDC,BTP,MX, } \\
\text { CULBTS,BTC,VS, } \\
\text { BTP,DC,CUL, } \\
\text { BTC,BTSBTS, } \\
\text { CAF,BC,VC, } \\
\text { BTCBTS, } \\
\text { BTC,VS }\end{array}$ & $1,2,3,4,5,6,7$ & & & \\
\hline \multicolumn{8}{|l|}{$\begin{array}{l}\text { Dermanura azteca } \\
\quad \text { (Andersen, 1906) }\end{array}$} \\
\hline $\begin{array}{l}\text { Dermanura azteca azteca } \\
\quad \text { (Andersen, 1906) }\end{array}$ & $\begin{array}{l}\text { MVO,PCP,SMO, } \\
\text { SMOC,SMS,VC }\end{array}$ & $\begin{array}{l}1,4,5,6 \\
8,10,11 \\
18,20,22 \\
27,28,30\end{array}$ & BCVS,BTS & $3,4,5,6$ & & & \\
\hline $\begin{array}{l}\text { Dermanura phaeotis Miller, } \\
\quad 1902\end{array}$ & $\begin{array}{l}\text { DIT,MVC, } \\
\text { PCG,PCP, } \\
\text { PCT,SMO, } \\
\text { SMOC,SMS }\end{array}$ & $\begin{array}{l}8,9,10,14 \\
11,17 \\
26,28\end{array}$ & $\begin{array}{l}\text { BC,BTC,BTS, } \\
\text { CUL,VS }\end{array}$ & $1,2,3,4$ & & & \\
\hline $\begin{array}{l}\text { Dermanura phaeotis nana } \\
\text { (Andersen, 1906) }\end{array}$ & $\begin{array}{l}\text { DIT,MVC,PCG, } \\
\text { PCP,SMS }\end{array}$ & $9,10,11,17,26,28$ & BTC,BTS & $1,2,3$ & & & \\
\hline $\begin{array}{l}\text { Dermanura phaeotis } \\
\text { palatinus (W. B. Davis, } \\
\text { 1970) }\end{array}$ & PCT & 10 & CUL & 1 & & & \\
\hline $\begin{array}{l}\text { Dermanura phaeotis phaeotis } \\
\quad \text { Miller, } 1902\end{array}$ & $\begin{array}{l}\text { DIT,MVC,PCG, } \\
\text { PCT,SMO,SMOC }\end{array}$ & $8,10,14,26,28$ & $\begin{array}{l}\text { BC,BTP,BTS, } \\
\text { BTC,CUL }\end{array}$ & $1,2,3$ & & & \\
\hline $\begin{array}{l}\text { Dermanura tolteca (De } \\
\text { Saussure, } 1860)\end{array}$ & $\begin{array}{l}\text { FT,MVC,MVO,PCG, } \\
\text { PCT,PCP,SMO, } \\
\text { SMOC,SMS,VC }\end{array}$ & $\begin{array}{l}2,7,8,9,10,11 \\
13,15,17,18,20 \\
22,26,27,30\end{array}$ & $\begin{array}{l}\text { BC,BTC,BTS,BTP, } \\
\text { DC,CUL,BMM,VS }\end{array}$ & $1,2,3,4,5,6$ & & & \\
\hline $\begin{array}{l}\text { Dermanura tolteca hespera } \\
\text { (W. B. Davis, 1970) }\end{array}$ & $\begin{array}{l}\text { FT,MVC,MVO, } \\
\text { PCG,PCT,PCP, } \\
\text { SMO,SMOC,SMS }\end{array}$ & $\begin{array}{l}10,11,13,17 \\
20,22,26,28,30\end{array}$ & BTC,VS,DC,CUL & $1,2,3,5,6$ & & & \\
\hline $\begin{array}{l}\text { Dermanura tolteca tolteca } \\
\quad \text { (De Saussure, } 1860)\end{array}$ & $\begin{array}{l}\text { PCG,PCP,SMO, } \\
\text { SMOC }\end{array}$ & $8,10,11,22,26$ & BC,BTP,BTS & $1,2,3,4,5,6$ & & & \\
\hline $\begin{array}{l}\text { Dermanura watsoni Thomas, } \\
\quad 1901\end{array}$ & PCG,SMOC,SMS & 10,17 & BTS,CAF,CUL & 1,2 & $\operatorname{Pr}$ & & \\
\hline $\begin{array}{l}\text { Enchisthenes hartii (Thomas, } \\
\text { 1892) }\end{array}$ & $\begin{array}{l}\text { PCG,PCT,PCP, } \\
\text { SMO,SMS }\end{array}$ & $\begin{array}{l}2,9,10,11 \\
14,22,26\end{array}$ & $\mathrm{BC}, \mathrm{BMM}$ & $1,2,3,4,5$ & $\operatorname{Pr}$ & & \\
\hline \multicolumn{8}{|l|}{ Centurio senex Gray, 1842} \\
\hline $\begin{array}{l}\text { Centurio senex senex Gray, } \\
1842\end{array}$ & $\begin{array}{l}\text { PCG,PCT,PCP, } \\
\text { SMO,SMOC,SMS }\end{array}$ & $\begin{array}{l}2,8,9,10,11 \\
17,21,22,26,27\end{array}$ & BC,BTS,CAF & $1,2,3,6$ & & & \\
\hline $\begin{array}{l}\text { Chiroderma salvini Dobson, } \\
1878\end{array}$ & $\begin{array}{l}\text { FT,MVO,PCG, } \\
\text { PCP,SMS }\end{array}$ & $\begin{array}{l}4,8,11,13,17 \\
20,25,26\end{array}$ & BC,BTP,CAF & $1,2,3$ & & & \\
\hline $\begin{array}{l}\text { Chiroderma salvini scopaeum } \\
\text { Handley, } 1966\end{array}$ & MVO,SMO,SMS & $8,13,25$ & BC,BТP,CAF & 3,5 & & & \\
\hline
\end{tabular}




\begin{tabular}{|c|c|c|c|c|c|c|c|}
\hline Lista sistemática & Subprovincia fisiográfica & Distrito & Vegetación & Altitud & $\mathrm{NOM}$ & IUCN & Cites \\
\hline \multicolumn{8}{|l|}{$\begin{array}{l}\text { Chiroderma villosum Peters, } \\
1860\end{array}$} \\
\hline $\begin{array}{l}\text { Chiroderma villosum jesupi J. } \\
\text { A. Allen, } 1900\end{array}$ & $\begin{array}{l}\text { MVO,PCG,PCT, } \\
\text { SMOC }\end{array}$ & $2,10,26,30$ & BC,BTP,VS & 1,6 & & & \\
\hline $\begin{array}{l}\text { Platyrrhinus helleri (Peters, } \\
\text { 1866) }\end{array}$ & $\begin{array}{l}\text { DIT,FT,MVO, } \\
\text { PCG,SMO,SMOC }\end{array}$ & $4,7,8,10,26$ & BTP,CUL,VS & $1,2,3,4$ & & & \\
\hline $\begin{array}{l}\text { Uroderma bilobatum Peters, } \\
1866\end{array}$ & $\begin{array}{l}\text { PCG,PCT,SMO, } \\
\text { SMOC }\end{array}$ & $10,21,26$ & DC & $1,2,4$ & & & \\
\hline $\begin{array}{l}\text { Uroderma bilobatum davisi } \\
\text { R. J. Baker y McDaniel, } \\
1972\end{array}$ & PCT,SMO & 10 & BTP,BTS,CUL & 1,2 & & & \\
\hline $\begin{array}{l}\text { Uroderma magnirostrum } \\
\text { Davis, } 1968\end{array}$ & PCT,SMO & 10 & BTP,BTS & 1,2 & & & \\
\hline $\begin{array}{l}\text { Vampyressa thyone Thomas, } \\
1909\end{array}$ & $\begin{array}{l}\text { FT,PCG,PCT,PCP, } \\
\text { SMOC,SMS }\end{array}$ & $2,10,11,17,22$ & BC,CAF,BMM,VS & $1,2,3$ & & & \\
\hline $\begin{array}{l}\text { Vampyrodes major G. M. } \\
\text { Allen, } 1908 \text { (Thomas, } \\
\text { 1889) }\end{array}$ & PCG,SMO,SMOC & $2,8,10,26$ & BTP,BTS,CUL & 1,3 & & & \\
\hline \multicolumn{8}{|l|}{$\begin{array}{l}\text { Sturnira hondurensis } \\
\text { Goodwin, } 1940\end{array}$} \\
\hline $\begin{array}{l}\text { Sturnira hondurensis } \\
\text { hondurensis Goodwin, } \\
1940\end{array}$ & $\begin{array}{l}\text { FT,MVC,MVO,PCG, } \\
\text { PCT,PCP,SMO, } \\
\text { SMOC,SMS,VC }\end{array}$ & $\begin{array}{l}1,4,6,8,10,11, \\
13,14,15,17,18, \\
20,22,24,25,26,27,30\end{array}$ & $\begin{array}{l}\text { BC,CAF,BTSBC, } \\
\text { BMM,BTC,BTP, } \\
\text { BTS,MX,CUL,VS }\end{array}$ & $1,2,3,4,5,6$ & & & \\
\hline $\begin{array}{l}\text { Sturnira parvidens Goldman, } \\
\quad 1917\end{array}$ & $\begin{array}{l}\text { DIT,FT,MVO,PCG, } \\
\text { PCT,PCP,SMO, } \\
\text { SMOC,SMS,VC }\end{array}$ & $\begin{array}{l}1,2,4,6,7,8,9 \\
10,11,13,14, \\
17,18,20,21, \\
22,26,27,28,30\end{array}$ & $\begin{array}{l}\text { BC,BTS,BC,CAF, } \\
\text { BTS,VSBTC,VS,DC, } \\
\text { MX,CUL,BMM }\end{array}$ & $1,2,3,4,5,6$ & & & \\
\hline \multicolumn{8}{|l|}{ Familia Vespertilionidae } \\
\hline $\begin{array}{l}\text { Bauerus dubiaquercus (Van } \\
\text { Gelder, 1959) }\end{array}$ & SMO & 22 & BTS,VS & 3 & & NT & \\
\hline $\begin{array}{l}\text { Myotis albescens (E. } \\
\text { Geoffroy Saint-Hilaire, } \\
1806)\end{array}$ & SMOC & 10 & BTP & 1 & $\operatorname{Pr}$ & & \\
\hline \multicolumn{8}{|l|}{$\begin{array}{l}\text { Myotis californicus (Audubon } \\
\text { y Bachman, 1842) }\end{array}$} \\
\hline $\begin{array}{l}\text { Myotis californicus } \\
\text { mexicanus (De Saussure, } \\
\text { 1860) }\end{array}$ & $\begin{array}{l}\text { FT,MVC,MVO,PCP, } \\
\text { SMO,SMOC,SMS,VC }\end{array}$ & $\begin{array}{l}1,4,6,8,10,11,14, \\
20,22,24,25,28,29,30\end{array}$ & $\mathrm{BC}, \mathrm{MX}$ & $1,2,3,4,5,6,7$ & & & \\
\hline \multicolumn{8}{|l|}{$\begin{array}{l}\text { Myotis fortidens Miller y G. } \\
\text { M. Allen, } 192 \text { MX }\end{array}$} \\
\hline $\begin{array}{l}\text { Myotis fortidens fortidens } \\
\quad \text { Miller y G. M. Allen, } 1928\end{array}$ & $\begin{array}{l}\text { DIT,MVC,MVO, } \\
\text { PCG,PCT,PCP,VC }\end{array}$ & $\begin{array}{l}1,9,10,11 \\
20,21,26,28\end{array}$ & BC,BTP,BTC,BTS,CUL & $1,2,4$ & & & \\
\hline \multicolumn{8}{|l|}{$\begin{array}{l}\text { Myotis keaysi J. A. Allen, } \\
1914\end{array}$} \\
\hline $\begin{array}{l}\text { Myotis keaysi pilosatibialis } \\
\quad \text { LaVal, } 1973\end{array}$ & $\begin{array}{l}\text { PCG,PCT,PCP, } \\
\text { SMO,SMOC,VC }\end{array}$ & $\begin{array}{l}8,10,11 \\
24,26,30\end{array}$ & BC,BMM,BTP,CUL & $1,3,4,5,6$ & & & \\
\hline \multicolumn{8}{|l|}{$\begin{array}{l}\text { Myotis nigricans (Schinz, } \\
\text { 1821) }\end{array}$} \\
\hline $\begin{array}{l}\text { Myotis nigricans nigricans } \\
\quad \text { (Schinz, 1821) }\end{array}$ & $\begin{array}{l}\text { DIT,MVO,PCG, } \\
\text { PCT,PCP,SMO,SMS }\end{array}$ & $\begin{array}{l}8,10,11 \\
14,20,26\end{array}$ & BC,BTS,BMM & $1,2,3,4,6$ & & & \\
\hline \multicolumn{8}{|l|}{$\begin{array}{l}\text { Myotis thysanodes Miller, } \\
1897\end{array}$} \\
\hline $\begin{array}{l}\text { Myotis thysanodes aztecus } \\
\text { Miller y G. M. Allen, } 1928\end{array}$ & $\begin{array}{l}\text { MVO,PCT,SMO, } \\
\text { SMS,VC }\end{array}$ & $\begin{array}{l}8,13,21 \\
29,30\end{array}$ & $\begin{array}{l}\text { BC,BTC,BTS, } \\
\text { BTP,CUL }\end{array}$ & $1,4,5,6$ & & & \\
\hline $\begin{array}{l}\text { Myotis velifer (J. A. Allen, } \\
\text { 1890) }\end{array}$ & & & $\mathrm{BC}$ & & & & \\
\hline $\begin{array}{l}\text { Myotis velifer velifer (J. A. } \\
\text { Allen, 1890) }\end{array}$ & $\begin{array}{l}\text { FT,MVC,MVO,PCT, } \\
\text { PCP,SMO,SMS,VC }\end{array}$ & $\begin{array}{l}1,8,11,12,13,17, \\
20,21,22,23,25,28,30\end{array}$ & BC,BTC,MX,CUL & $1,2,3,4,5,6$ & & & \\
\hline \multicolumn{8}{|l|}{$\begin{array}{l}\text { Perimyotis subflavus (F. } \\
\quad \text { Cuvier, 1832) }\end{array}$} \\
\hline $\begin{array}{l}\text { Perimyotis subflavus } \\
\quad \text { veraecrucis (Ward, 1891) }\end{array}$ & DIT & 10 & BTP & 1 & & & \\
\hline
\end{tabular}




\begin{tabular}{|c|c|c|c|c|c|c|c|}
\hline Lista sistemática & Subprovincia fisiográfica & Distrito & Vegetación & Altitud & NOM & IUCN & Cites \\
\hline \multicolumn{8}{|l|}{$\begin{array}{l}\text { Eptesicus brasiliensis } \\
\text { (Desmarest, 1819) }\end{array}$} \\
\hline $\begin{array}{l}\text { Eptesicus brasiliensis } \\
\text { brasiliensis (Desmarest, } \\
\text { 1819) }\end{array}$ & SMOC,SMS & $10,11,17,18$ & BTP & 2,4 & & & \\
\hline \multicolumn{8}{|l|}{$\begin{array}{l}\text { Eptesicus furinalis } \\
\text { (D’Orbigny y Gervais, } \\
\text { 1847) }\end{array}$} \\
\hline $\begin{array}{l}\text { Eptesicus furinalis gaumeri } \\
\quad \text { (J. A. Allen, 1897) }\end{array}$ & $\begin{array}{l}\text { MVO,PCG,PCP, } \\
\text { SMOC,SMS }\end{array}$ & $\begin{array}{l}7,10,11,13 \\
17,26\end{array}$ & BTP,BTS,CUL & $1,2,3,5$ & & & \\
\hline $\begin{array}{l}\text { Eptesicus fuscus (Palisot de } \\
\text { Beauvois, 1796) }\end{array}$ & $\begin{array}{l}\text { MVO,PCT,SMO, } \\
\text { SMS,VC }\end{array}$ & $\begin{array}{l}1,7,8,10,11 \\
13,20,29,30\end{array}$ & & $1,2,3,4,5,6$ & & & \\
\hline $\begin{array}{l}\text { Eptesicus fuscus miradorensis } \\
\quad(\mathrm{H} . \text { Allen, 1866) }\end{array}$ & $\begin{array}{l}\text { FT,MVC,MVO, } \\
\text { PCP,SMO, } \\
\text { SMS,VC }\end{array}$ & $\begin{array}{l}1,4,6,8,11,13 \\
17,20,22,23 \\
25,28,30\end{array}$ & BC,BTC,MX & $2,3,4,5,6$ & & & \\
\hline $\begin{array}{l}\text { Lasiurus blossevillii (Lesson, } \\
\text { 1826) }\end{array}$ & $\begin{array}{l}\text { MVO,PCP,PCT, } \\
\text { SMO,SMOC }\end{array}$ & $\begin{array}{l}4,6,7,8,10 \\
21,22,25,30\end{array}$ & $\begin{array}{l}\text { BC,BTC,BTP, } \\
\text { BTS,BE }\end{array}$ & $1,3,4,5,6$ & & & \\
\hline $\begin{array}{l}\text { Lasiurus blossevillii teliotis } \\
\quad \text { (H. Allen, 1891) }\end{array}$ & $\begin{array}{l}\text { MVO,PCP,PCT, } \\
\text { SMO,SMOC }\end{array}$ & $\begin{array}{l}4,6,7,8,10 \\
21,22,25,30\end{array}$ & $\begin{array}{l}\text { BC,BTC,BTP, } \\
\text { BTS,BE }\end{array}$ & $1,3,4,5,6$ & & & \\
\hline \multicolumn{8}{|l|}{$\begin{array}{l}\text { Lasiurus cinereus (Palisot de } \\
\text { Beauvois, 1796) }\end{array}$} \\
\hline $\begin{array}{l}\text { Lasiurus cinereus cinereus } \\
\quad \text { (Palisot de Beauvois, 1796) }\end{array}$ & $\begin{array}{l}\text { MVC,MVO,PCG, } \\
\text { PCT,PCP,SMO, } \\
\text { SMS,VC }\end{array}$ & $\begin{array}{l}1,6,7,8,10,13 \\
15,20,21,22,25,28\end{array}$ & $\begin{array}{l}\text { BC,BTS,BTC, } \\
\text { BE,VS }\end{array}$ & $1,2,3,4,5,6$ & & & \\
\hline \multicolumn{8}{|l|}{ Lasiurus ega (Gervais, 1856) } \\
\hline $\begin{array}{l}\text { Lasiurus ega panamensis } \\
\quad \text { (Thomas, 1901) }\end{array}$ & SMOC & 10 & BTP & 1 & & & \\
\hline \multicolumn{8}{|l|}{$\begin{array}{l}\text { Lasiurus intermedius } \mathrm{H} . \\
\quad \text { Allen, } 1862\end{array}$} \\
\hline $\begin{array}{l}\text { Lasiurus intermedius } \\
\quad \text { intermedius } \mathrm{H} \text {. Allen, } 1862\end{array}$ & $\begin{array}{l}\text { DIT,MVO,PCG,PCT, } \\
\text { PCP,SMO,SMS, } \\
\text { VC }\end{array}$ & $\begin{array}{l}1,6,8,10,21 \\
22,25,26,28\end{array}$ & $\begin{array}{l}\text { BC,BTP,VS, } \\
\text { CUL,BTC,BE }\end{array}$ & $1,3,4,5,6$ & & & \\
\hline $\begin{array}{l}\text { Lasiurus xanthinus (Thomas, } \\
\text { 1897) }\end{array}$ & PCG,PCT & 10,26 & BTP & 1 & & & \\
\hline $\begin{array}{l}\text { Rhogeessa alleni Thomas, } \\
189 \mathrm{MX}\end{array}$ & $\begin{array}{l}\text { FT,MVC,MVO, } \\
\text { SMO }\end{array}$ & $4,7,8,23,28$ & BTC,MX,BTS & $2,3,4,5$ & & & \\
\hline $\begin{array}{l}\text { Rhogeessa gracilis (Miller, } \\
\text { 1897) MX }\end{array}$ & FT,MVO,PCT,VC & $1,4,7,21,22$ & BC,MX,CUL & $1,2,3,4$ & & & \\
\hline \multicolumn{8}{|l|}{$\begin{array}{l}\text { Rhogeessa parvula } \mathrm{H} \text {. Allen, } \\
186 \mathrm{MX}\end{array}$} \\
\hline $\begin{array}{l}\text { Rhogeessa parvula parvula } \\
\text { H. Allen, } 186 \text { MX }\end{array}$ & $\begin{array}{l}\text { MVC,MVO,PCT, } \\
\text { PCP,SMO,SMOC, } \\
\text { SMS }\end{array}$ & $\begin{array}{l}7,10,11 \\
17,21,28\end{array}$ & $\begin{array}{l}\text { BTC,BTSBTC,DC, } \\
\text { CUL,BE,BTS,VS }\end{array}$ & $1,2,4$ & & & \\
\hline $\begin{array}{l}\text { Rhogeessa tumida H. Allen, } \\
\quad 1866\end{array}$ & $\begin{array}{l}\text { MVC,PCT,PCP, } \\
\text { SMO,SMOC,SMS }\end{array}$ & $10,17,21,28$ & BTC,CUL & 1,2 & & & \\
\hline \multicolumn{8}{|l|}{$\begin{array}{l}\text { Corynorhinus towsendii } \\
\text { (Cooper, 1837) }\end{array}$} \\
\hline $\begin{array}{l}\text { Corynorhinus towsendii } \\
\text { australis Handley, } 1955 \\
\text { Idionycteris phyllotis (G. M. } \\
\text { Allen, 1916) }\end{array}$ & $\begin{array}{l}\text { MVC,MVO,PCT, } \\
\text { SMO,VC }\end{array}$ & $1,4,7,21,24$ & BC,CUL,VS & $1,4,6$ & & & \\
\hline $\begin{array}{l}\text { Idionycteris phyllotis } \\
\text { phyllotis (G. M. Allen, } \\
\text { 1916) }\end{array}$ & FT & 4 & BTC & 2 & & & \\
\hline \multicolumn{8}{|l|}{ Orden Primates } \\
\hline \multicolumn{8}{|l|}{ Familia Atelidae } \\
\hline Ateles geoffroyi* Kuhl, 1820 & & & & & $\mathrm{P}$ & EN & II \\
\hline $\begin{array}{l}\text { Ateles geoffroyi vellerosus } \\
\text { Gray, } 1866\end{array}$ & $\begin{array}{l}\text { DIT,MVC,PCG,PCT, } \\
\text { PCP,SMO,SMOC,SMS }\end{array}$ & $\begin{array}{l}2,8,10,14 \\
21,24,26\end{array}$ & BTP,CUL & $1,2,3,4$ & & & \\
\hline $\begin{array}{l}\text { Alouatta palliata* (Gray, } \\
\text { 1849) }\end{array}$ & & & & & & & \\
\hline $\begin{array}{l}\text { Alouatta palliata mexicana } \\
\quad(\text { Gray, } 1849)\end{array}$ & PCG,SMOC & 10 & BTP & 1 & $\mathrm{P}$ & & I \\
\hline
\end{tabular}




\begin{tabular}{|c|c|c|c|c|c|c|}
\hline Lista sistemática & Subprovincia fisiográfica & Distrito & Vegetación & Altitud & NOM & IUC \\
\hline \multicolumn{7}{|l|}{ Orden Lagomorpha } \\
\hline \multicolumn{7}{|l|}{ Familia Leporidae } \\
\hline $\begin{array}{l}\text { Lepus callotis* J. A. Wagler, } \\
\quad 1830\end{array}$ & & & & & & NT \\
\hline $\begin{array}{l}\text { Lepus callotis callotis } \mathrm{J} . \text { A. } \\
\text { Wagler, } 183 \text { MX }\end{array}$ & MVO,VC & $1,3,7,19$ & CUL & $3,4,5$ & & \\
\hline $\begin{array}{l}\text { Lepus flavigularis* J. A. } \\
\text { Wagner, } 1844 \text { OAX }\end{array}$ & РCT,PCP & 10,21 & CUL,BTC & 1 & $\mathrm{P}$ & $\mathrm{EN}$ \\
\hline $\begin{array}{l}\text { Sylvilagus brasiliensis } \\
\quad \text { (Linnaeus, } 1758 \text { ) }\end{array}$ & SMO & 22 & & 5 & & \\
\hline $\begin{array}{l}\text { Sylvilagus brasiliensis truei } \\
\quad \text { (J. A. Allen, 1890) }\end{array}$ & PCG,SMO & $10,14,22,26$ & BTP,BTS,BC,CUL & 1,5 & & \\
\hline \multicolumn{7}{|l|}{$\begin{array}{l}\text { Sylvilagus cunicularius } \\
\quad \text { (Waterhouse, 1848) MX }\end{array}$} \\
\hline $\begin{array}{l}\text { Sylvilagus cunicularius } \\
\text { cunicularius (Waterhouse, } \\
\text { 1848) }\end{array}$ & $\begin{array}{l}\text { MVO,PCP,SMO, } \\
\text { VC }\end{array}$ & $1,3,8,9,11,14$ & BMM & $1,4,5,6$ & & \\
\hline $\begin{array}{l}\text { Sylvilagus floridanus (J. A. } \\
\text { Allen, 1890) }\end{array}$ & $\begin{array}{l}\text { MVC,MVO,PCT, } \\
\text { PCP,SMO,SMOC, } \\
\text { SMS,VC }\end{array}$ & $\begin{array}{l}1,6,7,8,10,13 \\
14,17,21,22,24 \\
25,26,29\end{array}$ & $\begin{array}{l}\text { BMM,BTC,BC, } \\
\text { CUL,BTS,VS }\end{array}$ & $1,4,5,6$ & & \\
\hline $\begin{array}{l}\text { Sylvilagus floridanus aztecus } \\
\text { (J. A. Allen, 1890) OAX }\end{array}$ & $\begin{array}{l}\text { MVC,PCT,PCP, } \\
\text { SMO,SMOC,SMS,VC }\end{array}$ & $\begin{array}{l}1,8,10,13 \\
17,21,24\end{array}$ & $\begin{array}{l}\text { BTC,BC,CUL, } \\
\text { BTS,VS }\end{array}$ & $1,2,4,5,6$ & & \\
\hline $\begin{array}{l}\text { Sylvilagus floridanus russatus } \\
\quad \text { (J. A. Allen, 1904) }\end{array}$ & $\mathrm{MVO}, \mathrm{SMO}, \mathrm{VC}$ & $1,7,14,22,24,26$ & BC,BMM & $1,4,5,7$ & & \\
\hline \multicolumn{7}{|l|}{ Orden Rodentia } \\
\hline $\begin{array}{r}\text { Glaucomys volans* } \\
\text { (Linnaeus, } 1758)\end{array}$ & $\begin{array}{l}\text { MVC,MVO,SMO, } \\
\text { SMS,PCT,VC }\end{array}$ & $\begin{array}{l}1,8,10,13 \\
21,25,28\end{array}$ & $\mathrm{BC}$ & $1,2,3,4,6$ & A & \\
\hline $\begin{array}{l}\text { Glaucomys volans goldmani } \\
\text { (Nelson, 1904) }\end{array}$ & PCT & 10 & $\mathrm{BC}$ & 1 & & \\
\hline $\begin{array}{l}\text { Glaucomys volans oaxacensis } \\
\text { Goodwin, } 1961\end{array}$ & $\begin{array}{l}\text { MVC,MVO,SMO, } \\
\text { SMS,VC }\end{array}$ & $\begin{array}{l}1,8,13 \\
21,25,28\end{array}$ & $\mathrm{BC}$ & $2,3,4,6$ & & \\
\hline \multicolumn{6}{|l|}{$\begin{array}{l}\text { Otospermophilus variegatus } \\
\text { (Erxleben, 1777) }\end{array}$} & \\
\hline $\begin{array}{l}\text { Otospermophilus variegatus } \\
\text { variegatus (Erxleben, } \\
\text { 1777) }\end{array}$ & MVO & $4,7,25$ & BTC & 4,5 & & \\
\hline $\begin{array}{l}\text { Sciurus aureogaster } \mathrm{F} . \\
\quad \text { Cuvier, } 1829\end{array}$ & $\begin{array}{l}\text { DIT,MVC,MVO, } \\
\text { PCG,PCT,PCP, } \\
\text { SMO,SMOC,SMS,VC }\end{array}$ & $\begin{array}{l}1,4,6,8,9,10 \\
11,13,14,17,18, \\
21,22,24,25,26, \\
27,28,29\end{array}$ & $\begin{array}{l}\text { BMM,BTS,BC,CAF, } \\
\text { CUL,BTC,BE,BTP, } \\
\text { VS,BTS }\end{array}$ & $1,2,3,4,5,6,7$ & & \\
\hline $\begin{array}{l}\text { Sciurus aureogaster } \\
\quad \text { aureogaster F. Cuvier, } \\
1829\end{array}$ & $\begin{array}{l}\text { DIT,PCG,PCT, } \\
\text { PCP,SMO,SMS }\end{array}$ & $\begin{array}{l}8,10,17,18 \\
21,22,26\end{array}$ & BC,BTP,BTS,VS & $1,2,3,5$ & & \\
\hline $\begin{array}{l}\text { Sciurus aureogaster } \\
\quad \text { nigrescens Bennett, } 1833\end{array}$ & $\begin{array}{l}\text { DIT,MVC,PCT,PCP, } \\
\text { SMO,SMOC,SMS,VC }\end{array}$ & $\begin{array}{l}1,4,8,10,11,13 \\
14,17,21,24,28,29\end{array}$ & $\begin{array}{l}\text { BMM,BTS,BC,CAF, } \\
\text { CUL,BTC,BE,BTP,VS }\end{array}$ & $1,2,3,4,5,6,7$ & & \\
\hline \multicolumn{6}{|l|}{ Sciurus deppei Peters, 1864} & \\
\hline $\begin{array}{l}\text { Sciurus deppei deppei Peters, } \\
\quad 1864\end{array}$ & $\begin{array}{l}\text { DIT,PCG,PCT, } \\
\text { PCP,SMO,SMOC }\end{array}$ & $\begin{array}{l}4,8,10,14 \\
21,24,27\end{array}$ & $\begin{array}{l}\text { BC,BMM,CUL, } \\
\text { BTC,BTP,BTS }\end{array}$ & $1,2,3,4,5,6,7$ & & \\
\hline \multicolumn{6}{|l|}{ Familia Geomyidae } & \\
\hline $\begin{array}{l}\text { Orthogeomys cuniculus } \\
\text { Elliot, } 1905 \text { OAX }\end{array}$ & $\mathrm{MVC}, \mathrm{PCT}$ & $10,21,28$ & BTC,BTS,CUL & 1,2 & A & \\
\hline $\begin{array}{l}\text { Orthogeomys grandis } \\
\text { (Thomas, 1893) }\end{array}$ & $\begin{array}{l}\text { MVO,MVC,PCT, } \\
\text { PCP,SMO,SMOC, } \\
\text { SMS,VC }\end{array}$ & $\begin{array}{l}1,6,8,9,10,11 \\
13,14,17,20 \\
21,24,28,29,30\end{array}$ & $\begin{array}{l}\text { BC,BE,BMM,BTC, } \\
\text { BTS,CAF,CUL }\end{array}$ & $1,2,3,4,5,6,7$ & & \\
\hline $\begin{array}{l}\text { Orthogeomys grandis alleni } \\
\text { Nelson y Goldman, } 1930\end{array}$ & MVO,PCP,SMS & $9,17,30$ & $\begin{array}{l}\text { BC,BTC,BTS, } \\
\text { BTP,VS }\end{array}$ & $1,3,5$ & & \\
\hline $\begin{array}{l}\text { Orthogeomys grandis } \\
\text { alvarezi Schaldach, } 1966 \\
\text { OAX }\end{array}$ & MVO,PCP,SMS & $11,13,20$ & BTC,BTS,CUL & $2,3,4,5$ & & \\
\hline $\begin{array}{l}\text { Orthogeomys grandis carbo } \\
\text { Goodwin, } 1956 \text { OAX }\end{array}$ & $\begin{array}{l}\text { MVC,PCT,PCP, } \\
\text { SMO,SMS }\end{array}$ & $14,21,28$ & BC,BTC,BTS,CUL & $1,2,4$ & & \\
\hline
\end{tabular}




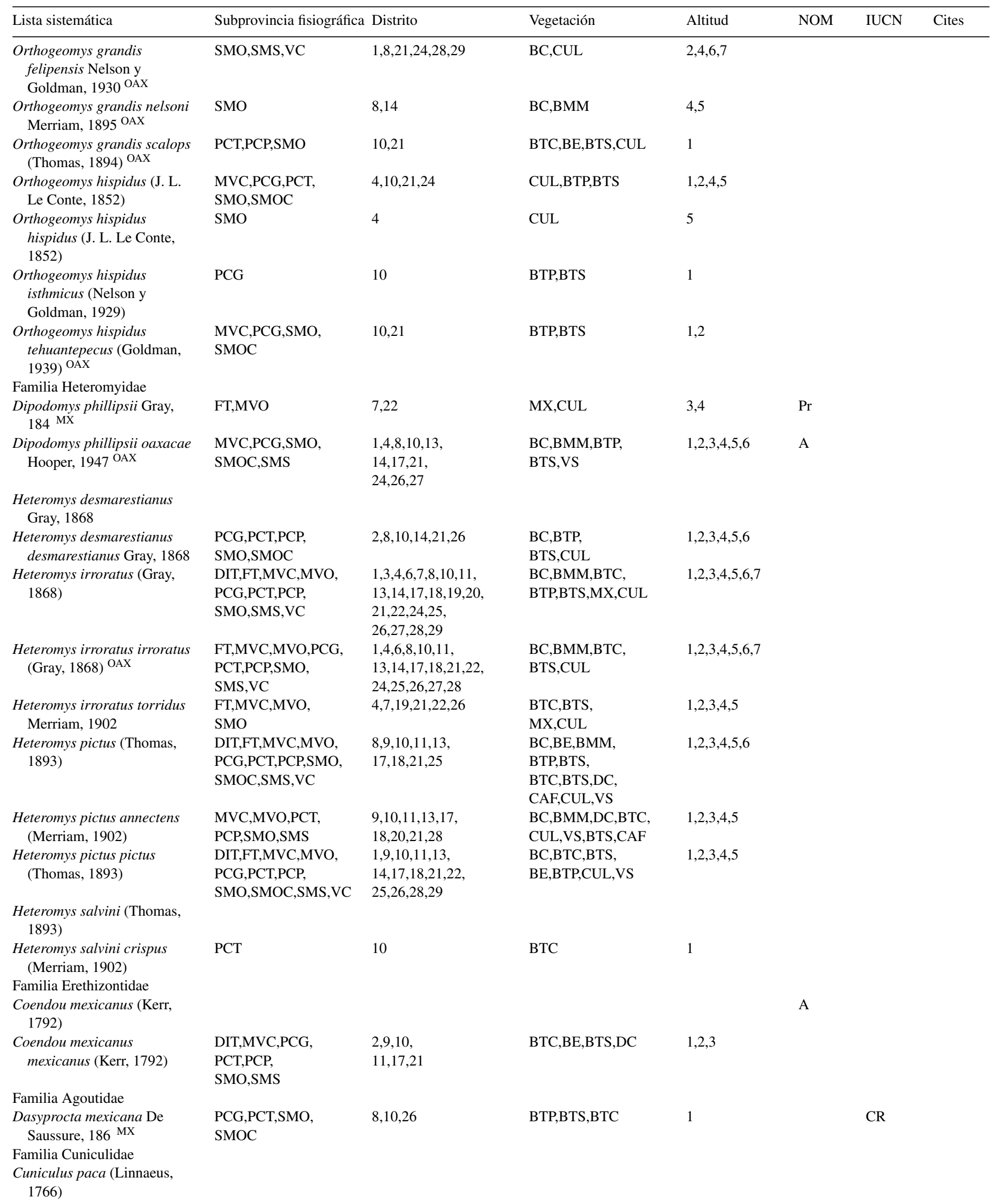




\begin{tabular}{|c|c|c|c|c|c|c|c|}
\hline Lista sistemática & Subprovincia fisiográfica & Distrito & Vegetación & Altitud & $\mathrm{NOM}$ & IUCN & Cites \\
\hline $\begin{array}{l}\text { Cuniculus paca nelsoni } \\
\quad \text { (Goldman, 1913) } \\
\text { Familia Cricetidae } \\
\text { Microtus mexicanus (De } \\
\quad \text { Saussure, 1861) }\end{array}$ & $\begin{array}{l}\text { FT,MVC,PCG, } \\
\text { SMO,SMOC }\end{array}$ & $\begin{array}{l}4,8,10,22 \\
24,26,27\end{array}$ & ВС,ВТРВС,ВТС & $1,2,3,4,5,6$ & & & \\
\hline $\begin{array}{l}\text { Microtus mexicanus } \\
\quad \text { fulviventer Merriam, } 189 \\
\text { MX }\end{array}$ & $\begin{array}{l}\text { MVO,PCT,PCP, } \\
\text { SMO,SMS,VC }\end{array}$ & $\begin{array}{l}1,4,8,11,12 \\
13,14,18,21,24 \\
25,26,27,29\end{array}$ & BCBMM,CUL & $1,2,3,4,5,6,7$ & & & \\
\hline $\begin{array}{l}\text { Microtus oaxacensis } \\
\text { Goodwin, } 1966^{\text {OAX }}\end{array}$ & SMO & 8 & $\mathrm{BC}$ & $3,4,5,6,7$ & A & EN & \\
\hline $\begin{array}{l}\text { Microtus quasiater (Coues, } \\
\text { 1874) MX }\end{array}$ & FT,SMO & 22 & $\mathrm{BC}, \mathrm{BMM}$ & $3,4,5$ & $\operatorname{Pr}$ & NT & \\
\hline $\begin{array}{l}\text { Microtus umbrosus Merriam, } \\
1898 \text { OAX }\end{array}$ & SMO & 14,27 & $\mathrm{BC}, \mathrm{BMM}$ & $4,5,6,7$ & $\operatorname{Pr}$ & EN & \\
\hline $\begin{array}{l}\text { Baiomys musculus (Merriam, } \\
\text { 1892) }\end{array}$ & $\begin{array}{l}\text { DIT,FT,MVC,MVO, } \\
\text { PCT,PCP,SMO, } \\
\text { SMOC,SMS,VC }\end{array}$ & $\begin{array}{l}1,4,5,6,7,9,10, \\
11,13,14,17,18, \\
20,21,22,23,24, \\
25,27,28,30\end{array}$ & $\begin{array}{l}\text { BCBC,BTP,BTS, } \\
\text { BTC,MX,VSBC, } \\
\text { BTC,BE,BTS,CUL }\end{array}$ & $\begin{array}{l}1,2,3,4 \\
5,6,7\end{array}$ & & & \\
\hline $\begin{array}{l}\text { Baiomys musculus infernatis } \\
\text { Hooper, } 1952^{\mathrm{MX}}\end{array}$ & FT,SMO,VC & $1,4,14,22,27$ & & $2,3,4$ & & & \\
\hline $\begin{array}{l}\text { Baiomys musculus pallidus } \\
\quad \text { Russell, } 1952^{\mathrm{MX}}\end{array}$ & $\begin{array}{l}\text { DIT,MVC,MVO, } \\
\text { PCT,PCP,SMO, } \\
\text { SMOC,SMS,SMS, } \\
\text { VC }\end{array}$ & $\begin{array}{l}1,5,6,7,9,10 \\
11,13,14,17,18 \\
20,21,23,24 \\
25,27,28,30\end{array}$ & & $1,2,3,4,5,6$ & & & \\
\hline $\begin{array}{l}\text { Scotinomys teguina (Alston, } \\
\text { 1877) }\end{array}$ & & & & & $\operatorname{Pr}$ & & \\
\hline $\begin{array}{l}\text { Scotinomys teguina teguina } \\
\quad \text { (Alston, 1877) }\end{array}$ & PCT,SMOC & 10 & BTP & 1,3 & & & \\
\hline $\begin{array}{l}\text { Hodomys alleni (Merriam, } \\
\text { 1892) MX }\end{array}$ & & & & & & & \\
\hline $\begin{array}{l}\text { Hodomys alleni vetulus } \\
\text { Merriam, } 189 \text { MX }\end{array}$ & FT,MVO & 7,22 & MX,CUL & 3,4 & & & \\
\hline $\begin{array}{l}\text { Neotoma mexicana Baird, } \\
1855\end{array}$ & $\begin{array}{l}\text { MVC,MVO,PCT, } \\
\text { PCP,SMO,SMOC, } \\
\text { SMS,VC }\end{array}$ & $\begin{array}{l}1,3,7,8,10, \\
11,13,14,17,18, \\
21,22,23,24,25,28\end{array}$ & $\begin{array}{l}\text { BC,BTC,BE,BTS, } \\
\text { BTP,CULBC,CUL, } \\
\text { VSBC,BMM }\end{array}$ & $1,2,3,4,5,6$ & & & \\
\hline $\begin{array}{l}\text { Neotoma mexicana isthmica } \\
\text { Goldman, } 1904^{\mathrm{MX}}\end{array}$ & $\begin{array}{l}\text { MVC,MVO,PCT, } \\
\text { PCP,SMO,SMS,VC }\end{array}$ & $\begin{array}{l}1,3,11,17 \\
21,22,24\end{array}$ & & $1,2,3,4,5$ & & & \\
\hline $\begin{array}{l}\text { Neotoma mexicana parvidens } \\
\text { Goldman, } 190 \mathrm{MX}\end{array}$ & PCP,SMS & $11,13,17$ & & $2,3.4 .5$ & & & \\
\hline $\begin{array}{l}\text { Neotoma mexicana picta } \\
\text { Goldman, } 1904^{\mathrm{MX}}\end{array}$ & $\begin{array}{l}\text { MVO,PCT,PCP, } \\
\text { SMS }\end{array}$ & $\begin{array}{l}10,13,17,18 \\
23,25,28\end{array}$ & & $1,4,5,6$ & & & \\
\hline $\begin{array}{l}\text { Neotoma mexicana tropicalis } \\
\text { Goldman, } 190 \mathrm{MX}\end{array}$ & SMO,SMOC & $8,10,14$ & & $3,4,5$ & & & \\
\hline $\begin{array}{l}\text { Habromys chinanteco } \\
\text { (Robertson y Musser, } \\
\text { 1976) OAX }\end{array}$ & SMO & 8 & $\mathrm{BC}$ & 5,6 & & $\mathrm{CR}$ & \\
\hline $\begin{array}{l}\text { Habromys ixtlani (Goodwin, } \\
\text { 1964) OAX }\end{array}$ & SMO, VC & $1,4,8,26$ & BC,BMM & $3,4,5,6,7$ & & $\mathrm{CR}$ & \\
\hline $\begin{array}{l}\text { Habromys lepturus (Merriam, } \\
\text { 1898) OAX }\end{array}$ & SMO & $8,14,27$ & $\mathrm{BC}, \mathrm{BMM}$ & $3,4,5,6,7$ & & $\mathrm{CR}$ & \\
\hline $\begin{array}{l}\text { Habromys simulatus } \\
\text { (Osgood, 1904) } \mathrm{MX}\end{array}$ & SMO & 22 & $\mathrm{BC}, \mathrm{BMM}$ & 5 & $\operatorname{Pr}$ & $\mathrm{CR}$ & \\
\hline $\begin{array}{l}\text { Megadontomys cryophilus } \\
\text { (Musser, 1964) OAX }\end{array}$ & SMO & $4,8,27$ & $\mathrm{BC}, \mathrm{BMM}$ & $2,3,4,5,6,7$ & A & EN & \\
\hline $\begin{array}{l}\text { Megadontomys nelsoni } \\
\text { (Merriam, 1898) MX }\end{array}$ & FT,SMO & 4,22 & BMM & $2,3,4,5,6$ & A & EN & \\
\hline $\begin{array}{l}\text { Megadontomys thomasi } \\
\text { (Merriam, 1898) MX }\end{array}$ & SMS & 18 & $\mathrm{BC}$ & 2 & $\operatorname{Pr}$ & EN & \\
\hline $\begin{array}{l}\text { Peromyscus aztecus (De } \\
\text { Saussure, 1860) }\end{array}$ & $\begin{array}{l}\text { DIT,FT,MVC,MVO, } \\
\text { PCG,PCT,PCP, } \\
\text { SMO,SMOC,SMS,VC }\end{array}$ & $\begin{array}{l}1,4,8,9,10,11, \\
13,14,17,18, \\
20,21,22,24, \\
25,26,27,28,29,30\end{array}$ & $\begin{array}{l}\text { BTS,BC,CAF,BTS, } \\
\text { BTC,VS,BC,CAFBMM, } \\
\text { BTCBC,BMM,BTP, } \\
\text { BTS,MX,CUL }\end{array}$ & $1,2,3,4,5,6$ & & & \\
\hline
\end{tabular}




\begin{tabular}{|c|c|c|c|c|c|c|c|}
\hline Lista sistemática & Subprovincia fisiográfica & Distrito & Vegetación & Altitud & $\mathrm{NOM}$ & IUCN & Cites \\
\hline $\begin{array}{l}\text { Peromyscus aztecus evides } \\
\text { Osgood, } 190 \text { MX }\end{array}$ & $\begin{array}{l}\text { MVC,MVO,PCG, } \\
\text { PCT,PCP,SMO, } \\
\text { SMOC,SMS }\end{array}$ & $\begin{array}{l}8,9,10,11,13 \\
14,17,18,20 \\
21,28\end{array}$ & & $1,2,3,4,5,6$ & & & \\
\hline $\begin{array}{l}\text { Peromyscus aztecus } \\
\quad \text { oaxacensis Merriam, } 1898\end{array}$ & $\begin{array}{l}\text { FT,MVC,PCT, } \\
\text { SMO,SMOC,SMS,VC }\end{array}$ & $\begin{array}{l}1,4,8,10,11 \\
14,17,21,22 \\
26,27,29\end{array}$ & & $1,2,3,4,5,6$ & & & \\
\hline \multicolumn{8}{|l|}{$\begin{array}{l}\text { Peromyscus beatae Thomas, } \\
190 \text { MX }\end{array}$} \\
\hline $\begin{array}{l}\text { Peromyscus beatae beatae } \\
\text { Thomas, } 190 \mathrm{MX}\end{array}$ & $\begin{array}{l}\text { MVC,PCT,SMO, } \\
\text { SMS }\end{array}$ & $8,11,13,21,28$ & BC,BTC,BTS,CUL & $1,2,3,4,5,6,7$ & & & \\
\hline $\begin{array}{l}\text { Peromyscus difficilis (J. A. } \\
\text { Allen, 1891) }\end{array}$ & $\begin{array}{l}\text { MVO,SMO,SMS, } \\
\text { VC }\end{array}$ & $\begin{array}{l}1,3,6,8,13,14 \\
15,17,23,24,25 \\
26,27,29\end{array}$ & BC,BMMCUL & $1,3,4,5,6,7,8$ & & & \\
\hline $\begin{array}{l}\text { Peromyscus difficilis amplus } \\
\text { Osgood, } 190 \text { MX }\end{array}$ & MVO,SMO & $3,15,23,25,26$ & & 1,5 & & & \\
\hline $\begin{array}{l}\text { Peromyscus difficilis } \\
\quad \text { felipensis Merriam, } 189 \\
\text { MX }\end{array}$ & $\begin{array}{l}\text { MVO,SMO,SMS, } \\
\text { VC }\end{array}$ & $1,5,8,13,24,25$ & & $4,5,6,7$ & & & \\
\hline $\begin{array}{l}\text { Peromyscus furvus } \mathrm{J} \text {. A. Allen } \\
\text { y Chapman, } 189 \text { MX }\end{array}$ & SMO & 8,22 & BC,BMM,BTS,CUL & $3,4,5,6$ & & & \\
\hline $\begin{array}{l}\text { Peromyscus gratus Merriam, } \\
189 \text { MX }\end{array}$ & $\begin{array}{l}\text { MVC,MVO,SMO, } \\
\text { SMS,VC }\end{array}$ & $\begin{array}{l}1,7,8,13,22,23 \\
24,25,26,28\end{array}$ & BC,BMM,CUL & $1,2,3,4,5,6$ & & & \\
\hline $\begin{array}{l}\text { Peromyscus gratus zapotecae } \\
\text { Hooper, } 195 \text { MX }\end{array}$ & $\begin{array}{l}\text { MVC,MVO,SMO, } \\
\text { SMS }\end{array}$ & $7,8,13,22,24,25,26$ & & $1,2,3,4,5,6$ & & & \\
\hline $\begin{array}{l}\text { Peromyscus leucopus } \\
\text { (Rafinesque, 1818) }\end{array}$ & $\begin{array}{l}\text { FT,MVC,PCG, } \\
\text { PCT,SMO,SMOC,SMS }\end{array}$ & $\begin{array}{l}4,6,8,10,13 \\
14,21,22\end{array}$ & $\begin{array}{l}\text { BC,BTC,BTS,MX, } \\
\text { CUL,VSBTP,BTS, } \\
\text { CULBC,CUL }\end{array}$ & $1,2,3,4,5$ & & & \\
\hline $\begin{array}{l}\text { Peromyscus leucopus affinis } \\
\quad \text { (J. A. Allen, 1891) MX }\end{array}$ & FT,MVC,PCT,SMO & $\begin{array}{l}4,6,8 \\
10,21,22\end{array}$ & & $1,2,3,4,5$ & & & \\
\hline $\begin{array}{l}\text { Peromyscus leucopus } \\
\quad \text { incensus Goldman, } 194 \text { MX }\end{array}$ & FT,PCG,SMO & $4,10,14$ & & 1,2 & & & \\
\hline $\begin{array}{l}\text { Peromyscus leucopus } \\
\quad \text { lachiguiriensis Goodwin, } \\
1956 \text { OAX }\end{array}$ & SMS & 13 & & $1,4,5$ & & & \\
\hline $\begin{array}{l}\text { Peromyscus levipes Merriam, } \\
189 \text { MX }\end{array}$ & $\begin{array}{l}\text { FT,MVC,MVO,PCG, } \\
\text { PCT,PCP,SMO, } \\
\text { SMOC,SMS,VC }\end{array}$ & $\begin{array}{l}1,3,4,6,8,10,11 \\
13,14,15,17,18,20 \\
21,22,25,26,27,28\end{array}$ & $\begin{array}{l}\text { BC,BC,BMM,BTS, } \\
\text { BTC,BTP, } \\
\text { CULBMM,VS }\end{array}$ & $1,2,3,4,5,6,7$ & & & \\
\hline $\begin{array}{l}\text { Peromyscus levipes levipes } \\
\text { Merriam, } 189 \mathrm{MX}\end{array}$ & $\begin{array}{l}\text { FT,MVC,MVO,PCG, } \\
\text { PCT,PCP,SMO, } \\
\text { SMOC,SMS,VC }\end{array}$ & $\begin{array}{l}1,3,6,8,10,11 \\
13,15,17,18,20,21 \\
22,25,26,27,28\end{array}$ & & $1,2,3,4,5,6,7$ & & & \\
\hline $\begin{array}{l}\text { Peromyscus maniculatus } \\
\text { (Wagner, 1845) }\end{array}$ & $\begin{array}{l}\text { FT,MVC,MVO, } \\
\text { PCP,SMO,VC }\end{array}$ & $\begin{array}{l}1,3,4,7,8,11 \\
15,20,24,25,26,27\end{array}$ & $\begin{array}{l}\text { BC,BTC,BTS, } \\
\text { MX,CUL }\end{array}$ & $1,2,3,4,5$ & & & \\
\hline $\begin{array}{l}\text { Peromyscus maniculatus } \\
\text { fulvus Osgood, } 190 \mathrm{MX}\end{array}$ & $\begin{array}{l}\text { FT,MVC,MVO, } \\
\text { PCP,SMO,VC }\end{array}$ & $\begin{array}{l}1,3,4,7,8,11 \\
15,20,24,25,26\end{array}$ & & $1,2,3,4,5$ & & & \\
\hline $\begin{array}{l}\text { Peromyscus megalops } \\
\text { Merriam, } 189 \text { MX }\end{array}$ & $\begin{array}{l}\text { MVC,MVO,PCP, } \\
\text { SMO,SMS,VC }\end{array}$ & $\begin{array}{l}8,11,13,17,18 \\
20,22,27,28,29\end{array}$ & $\begin{array}{l}\text { BTS,BC,CAFBC, } \\
\text { BMM,BTS,CUL, } \\
\text { VSBC,BTP, } \\
\text { BTS, VS }\end{array}$ & $1,2,3,4,5,6$ & & & \\
\hline $\begin{array}{l}\text { Peromyscus megalops auritus } \\
\text { Merriam, } 189 \text { MX }\end{array}$ & $\begin{array}{l}\text { MVO,SMO,SMS, } \\
\text { VC }\end{array}$ & $\begin{array}{l}8,11,18 \\
22,27,29\end{array}$ & & $1,3,4,5$ & & & \\
\hline $\begin{array}{l}\text { Peromyscus megalops } \\
\quad \text { megalops Merriam, } 189 \\
\text { MX }\end{array}$ & $\begin{array}{l}\text { MVC,MVO,PCP, } \\
\text { SMO,SMS }\end{array}$ & $\begin{array}{l}8,11,13 \\
17,20,28\end{array}$ & & $2,3,4,5,6$ & & & \\
\hline $\begin{array}{l}\text { Peromyscus melanocarpus } \\
\text { Osgood, } 1904 \text { OAX }\end{array}$ & PCG,SMO & $\begin{array}{l}4,8,14,22 \\
26,27\end{array}$ & $\begin{array}{l}\text { BTS,BC, } \\
\text { BMM,BTP }\end{array}$ & $\begin{array}{l}1,2,3,4 \\
5,6,7\end{array}$ & & EN & \\
\hline $\begin{array}{l}\text { Peromyscus melanophrys } \\
\quad \text { (Coues, 1874) } \mathrm{MX}\end{array}$ & $\begin{array}{l}\text { DIT,FT,MVC,MVO, } \\
\text { PCT,PCP,SMO, } \\
\text { SMS,VC }\end{array}$ & $\begin{array}{l}1,3,4,6,7,8,9,10 \\
11,13,14,17,20,21 \\
22,23,26,27,28\end{array}$ & $\begin{array}{l}\text { BTS,BC,BTC, } \\
\text { MX,CUL,VS }\end{array}$ & $1,2,3,4,5,6$ & & & \\
\hline $\begin{array}{l}\text { Peromyscus melanophrys } \\
\text { melanophrys (Coues, } \\
\text { 1874) }\end{array}$ & $\begin{array}{l}\text { DIT,FT,MVC,MVO, } \\
\text { PCT,PCP,SMO, } \\
\text { SMS,VC }\end{array}$ & $\begin{array}{l}4,6,7,8,9,10,13 \\
14,17,20,21 \\
22,23,24,26,27,28\end{array}$ & & $1,2,3,4,5,6$ & & & \\
\hline $\begin{array}{l}\text { Peromyscus melanotis J. A. } \\
\text { Allen y Chapman, } 1897\end{array}$ & SMO & 8 & $\mathrm{BC}$ & 7 & & & \\
\hline
\end{tabular}




\begin{tabular}{|c|c|c|c|c|c|c|c|}
\hline Lista sistemática & Subprovincia fisiográfica & Distrito & Vegetación & Altitud & NOM & IUCN & Cites \\
\hline $\begin{array}{l}\text { Peromyscus melanurus } \\
\text { Osgood, } 1909 \text { OAX }\end{array}$ & MVO,PCP,SMS & $11,13,17,25$ & $\begin{array}{l}\text { BC,BMMBTP, } \\
\text { BTS,CUL }\end{array}$ & $2,3,4,5,6$ & & EN & \\
\hline $\begin{array}{l}\text { Peromyscus mexicanus (De } \\
\text { Saussure, 1860) }\end{array}$ & $\begin{array}{l}\text { DIT,FT,MVC,MVO, } \\
\text { PCG,PCT,PCP, } \\
\text { SMO,SMOC,SMS,VC }\end{array}$ & $\begin{array}{l}1,2,4,8,9,10,11, \\
13,14,17,18,20 \\
21,22,25,26,27,28\end{array}$ & $\begin{array}{l}\text { BTS,BC,BMM, } \\
\text { BC,BTC,BTS,CUL, } \\
\text { VSCAF,BTPBTP, } \\
\text { BTS,BTC, } \\
\text { CULBC,BMM, } \\
\text { BTS,BTP, } \\
\text { BMM,BC,VS }\end{array}$ & $1,2,3,4,5,6,7$ & & & \\
\hline $\begin{array}{l}\text { Peromyscus mexicanus } \\
\text { angelensis Osgood, } 1904 \\
\text { OAX }\end{array}$ & $\begin{array}{l}\text { MVC,PCT,PCP, } \\
\text { SMO,SMOC,SMS,VC }\end{array}$ & $\begin{array}{l}9,10,11,13 \\
17,18,21,28,30\end{array}$ & & $1,2,3,4,5$ & & & \\
\hline $\begin{array}{l}\text { Peromyscus mexicanus } \\
\text { azulensis Goodwin, } 1956 \\
\text { OAX }\end{array}$ & SMS & 13 & & 5 & & & \\
\hline $\begin{array}{l}\text { Peromyscus mexicanus } \\
\text { mexicanus (De Saussure, } \\
\text { 1860) MX }\end{array}$ & $\begin{array}{l}\text { DIT,MVC,PCG, } \\
\text { PCT,PCP,SMO, } \\
\text { SMOC,SMS }\end{array}$ & $\begin{array}{l}10,11,13,14 \\
17,21,22,26\end{array}$ & & $1,2,3,4,5$ & & & \\
\hline $\begin{array}{l}\text { Peromyscus mexicanus } \\
\text { putlaensis Goodwin, } 1964 \\
\text { OAX }\end{array}$ & MVP,SMS & $17,18,25$ & & $3,4,5$ & & & \\
\hline $\begin{array}{l}\text { Peromyscus mexicanus } \\
\text { tehuantepecus Merriam, } \\
1898 \text { OAX }\end{array}$ & PCT & 21 & & 1 & & & \\
\hline $\begin{array}{l}\text { Peromyscus mexicanus } \\
\quad \text { totontepecus Merriam, } 189 \\
\text { MX }\end{array}$ & $\begin{array}{l}\text { FT,MVC,PCG, } \\
\text { PCT,SMO,SMS }\end{array}$ & $\begin{array}{l}2,4,8,10,13,14 \\
21,22,26,27\end{array}$ & & $1,2,3,4,5,6$ & & & \\
\hline $\begin{array}{l}\text { Reithrodontomys fulvescens J. } \\
\quad \text { A Allen, } 1894\end{array}$ & $\begin{array}{l}\text { FT,MVC,MVO,PCT, } \\
\text { PCP,SMO,SMS,VC }\end{array}$ & $\begin{array}{l}1,4,6,7,8,9,10,11, \\
13,14,15,17,18,20, \\
21,22,23,24,25, \\
26,27,28,29,30\end{array}$ & $\begin{array}{l}\text { BC,BTS,CAFBTC, } \\
\text { VS,BC,BTP, } \\
\text { CULBC,MX, } \\
\text { CUL,BTS }\end{array}$ & $\begin{array}{l}1,2,3,4 \\
5,6,7\end{array}$ & & & \\
\hline $\begin{array}{l}\text { Reithrodontomys fulvescens } \\
\text { amoenus (Elliot, 1905) }^{\mathrm{MX}}\end{array}$ & $\mathrm{PCT}$ & 10 & & 1 & & & \\
\hline $\begin{array}{l}\text { Reithrodontomys fulvescens } \\
\text { fulvescens J. A Allen, } 1894\end{array}$ & MVO,SMO & 8,18 & & 5,6 & & & \\
\hline $\begin{array}{l}\text { Reithrodontomys fulvescens } \\
\text { helvolus Merriam, } 190 \mathrm{MX}\end{array}$ & $\begin{array}{l}\text { FT,MVC,MVO,PCP, } \\
\text { SMO,SMS,VC }\end{array}$ & $\begin{array}{l}1,4,5,7,8,9,11 \\
13,14,15,17,21,22, \\
23,24,25,26,27\end{array}$ & & $1,2,3,4,5,6$ & & & \\
\hline $\begin{array}{l}\text { Reithrodontomys fulvescens } \\
\text { infernatis Hooper, } 195 \text { MX }\end{array}$ & FT,SMO & $4,8,22$ & & $2,3,4$ & & & \\
\hline $\begin{array}{l}\text { Reithrodontomys fulvescens } \\
\text { mustelinus A. H. Howell, } \\
191 \text { MX }\end{array}$ & PCP,SMS & $9,11,17$ & & $1,2,3$ & & & \\
\hline $\begin{array}{l}\text { Reithrodontomys megalotis } \\
\quad \text { (Baird, 1857) }\end{array}$ & $\begin{array}{l}\text { MVO,PCP,SMO, } \\
\text { SMS,VC }\end{array}$ & $\begin{array}{l}1,8,11,13,14 \\
17,18,19,20,22,23\end{array}$ & $\begin{array}{l}\text { BCVS,BC,BMM, } \\
\text { BTS,BTP,CUL }\end{array}$ & $1,2,3,4,5,6,7$ & & & \\
\hline $\begin{array}{l}\text { Reithrodontomys megalotis } \\
\text { alticola Merriam, } 190 \text { MX }\end{array}$ & $\begin{array}{l}\text { MVO,PCP,SMO, } \\
\text { SMS,VC }\end{array}$ & $\begin{array}{l}1,8,11,13,14 \\
18,19,22,23\end{array}$ & & $\begin{array}{l}1,2,3,4 \\
5,6,7\end{array}$ & & & \\
\hline $\begin{array}{l}\text { Reithrodontomys mexicanus } \\
\text { (De Saussure, 1860) }\end{array}$ & $\begin{array}{l}\text { MVO,PCT,PCP, } \\
\text { SMO,SMOC, } \\
\text { SMS,VC }\end{array}$ & $\begin{array}{l}1,8,10,11,13 \\
14,17,18,21 \\
22,27\end{array}$ & $\begin{array}{l}\text { BMMVS,BC,BMM, } \\
\text { BTC,BTSBTC,BTS }\end{array}$ & $\begin{array}{l}1,2,3,4 \\
5,6,7\end{array}$ & & & \\
\hline $\begin{array}{c}\text { Reithrodontomys mexicanus } \\
\text { scansor Hooper, } 195 \text { MX }\end{array}$ & PCT,SMO,SMOC & & 8,10 & $1,3,4,5,6$ & & & \\
\hline $\begin{array}{l}\text { Reithrodontomys microdon } \\
\text { Merriam, } 1901\end{array}$ & & & & & & & \\
\hline $\begin{array}{l}\text { Reithrodontomys microdon } \\
\text { albilabris Merriam, } 1901 \\
\text { OAX }\end{array}$ & SMO,VC & $1,4,8,22,26$ & $\begin{array}{l}\text { BC,BMM,BTS, } \\
\text { CUL,VS }\end{array}$ & $3,4,5,6,7$ & A & & \\
\hline $\begin{array}{l}\text { Reithrodontomys sumichrasti } \\
\quad \text { (De Saussure, 1860) }\end{array}$ & $\begin{array}{l}\text { MVC,MVO,PCG, } \\
\text { PCT,PCP,SMO, } \\
\text { SMS,VC }\end{array}$ & $\begin{array}{l}1,4,8,9,10,11 \\
12,13,14,17 \\
18,21,22,24,26 \\
27,28,29\end{array}$ & $\begin{array}{l}\text { BCBC,BTC,BTS, } \\
\text { BTP,CUL,VSBC, } \\
\text { BMM,CUL }\end{array}$ & $1,2,3,4,5,6,7$ & & & \\
\hline $\begin{array}{l}\text { Reithrodontomys sumichrasti } \\
\text { luteolus A. H. Howell, } \\
1914\end{array}$ & $\begin{array}{l}\text { MVC,MVO,PCP, } \\
\text { SMO,SMS,VC }\end{array}$ & $\begin{array}{l}1,9,11,12,13 \\
17,18,21,22,28\end{array}$ & & $1,2,3,4,5,6$ & & & \\
\hline
\end{tabular}




\begin{tabular}{|c|c|c|c|c|c|c|c|}
\hline Lista sistemática & Subprovincia fisiográfica & Distrito & Vegetación & Altitud & NOM & IUCN & Cites \\
\hline $\begin{array}{l}\text { Reithrodontomys sumichrasti } \\
\text { sumichrasti (De Saussure, } \\
\text { 1860) MX }\end{array}$ & MVC,PCP,SMO,VC & $\begin{array}{l}1,4,8,14 \\
22,24,26,27\end{array}$ & & $1,3,4,5,6$ & & & \\
\hline $\begin{array}{l}\text { Oligoryzomys fulvescens (De } \\
\text { Saussure, 1860) }\end{array}$ & $\begin{array}{l}\text { FT,MVC,MVO,PCG, } \\
\text { PCT,PCP,SMO, } \\
\text { SMOC,SMS,VC }\end{array}$ & $\begin{array}{l}1,4,8,10,11,13 \\
14,17,20,21,22,26,27\end{array}$ & $\begin{array}{l}\text { BCBMM,BTP, } \\
\text { BTC,MX,CUL, } \\
\text { BTS,BC,BMM }\end{array}$ & $1,2,3,4,5,6$ & & & \\
\hline $\begin{array}{l}\text { Oligoryzomys fulvescens } \\
\text { fulvescens (De Saussure, } \\
\text { 1860) }\end{array}$ & $\begin{array}{l}\text { FT,MVC,PCG,PCT, } \\
\text { SMO,SMS,VC }\end{array}$ & $\begin{array}{l}1,4,8,10,11,13 \\
14,21,22,26,27\end{array}$ & & $1,2,3,4,5,6$ & & & \\
\hline $\begin{array}{l}\text { Oligoryzomys fulvescens } \\
\text { lenis (Goldman, 1915) } \mathrm{MX}\end{array}$ & $\begin{array}{l}\text { MVO,PCP,SMO, } \\
\text { SMOC,SMS }\end{array}$ & $\begin{array}{l}10,11,13 \\
17,20,26\end{array}$ & & $1,2,3,4,5,6$ & & & \\
\hline $\begin{array}{l}\text { Oligoryzomys fulvescens } \\
\text { pacificus (Hooper, 1952) }\end{array}$ & PCT & 10 & & 1 & & & \\
\hline $\begin{array}{l}\text { Oryzomys alfaroi (J. A. } \\
\text { Allen, 1891) }\end{array}$ & $\begin{array}{l}\text { MVC,MVO,PCT,PCP, } \\
\text { SMO,SMOC,SMS, } \\
\text { VC }\end{array}$ & $\begin{array}{l}8,10,11,13,14 \\
17,20,21,22 \\
24,26,27\end{array}$ & $\begin{array}{l}\text { ВCBTP,BTSBMM, } \\
\text { BC,BTS }\end{array}$ & $1,2,3,4,5,6,7$ & & & \\
\hline $\begin{array}{l}\text { Oryzomys alfaroi gloriaensis } \\
\text { Goodwin, } 1956 \text { OAX }\end{array}$ & SMOC & 10 & & $2,3,4$ & & & \\
\hline $\begin{array}{l}\text { Oryzomys alfaroi palatinus } \\
\text { Merriam, } 190 \text { MX }\end{array}$ & PCT,SMO,SMOC & $8,10,22$ & & $1,3,4,5,6$ & $\operatorname{Pr}$ & & \\
\hline $\begin{array}{l}\text { Oryzomys chapmani Thomas, } \\
189 \text { MX }\end{array}$ & $\begin{array}{l}\text { FT,MVC,MVO,PCG, } \\
\text { PCT,PCP,SMO,SMS }\end{array}$ & $\begin{array}{l}8,10,11,13,14 \\
17,18,20,21,22,26,27\end{array}$ & $\begin{array}{l}\text { BC,BMM,BTSCULBC, } \\
\text { BMMBMM,BTP,BC, } \\
\text { BTS,BTC,CULBC, } \\
\text { BMM,CUL }\end{array}$ & $1,2,3,4,5,6,7$ & & & \\
\hline $\begin{array}{l}\text { Oryzomys chapmani caudatus } \\
\text { Merriam, } 1901 \text { OAX }\end{array}$ & SMO & 8,14 & & $3,4,5,6$ & & & \\
\hline $\begin{array}{l}\text { Oryzomys chapmani } \\
\text { chapmani Thomas, } 189 \text { MX }\end{array}$ & $\begin{array}{l}\text { MVC,MVO,PCG, } \\
\text { SMO }\end{array}$ & $\begin{array}{l}8,10,14,20 \\
21,22,26,27\end{array}$ & & $1,2,3,4,5,6,7$ & & & \\
\hline $\begin{array}{l}\text { Oryzomys couesi (Alston, } \\
\text { 1877) }\end{array}$ & $\begin{array}{l}\text { DIT,FT,MVC,PCG, } \\
\text { PCT,PCP,SMO, } \\
\text { SMOC,SMS,VC }\end{array}$ & $\begin{array}{l}1,4,6,8,9,10,11 \\
13,14,17,21 \\
22,24,26,27\end{array}$ & $\begin{array}{l}\text { BC,BTS,CAF,MX, } \\
\text { CUL,PASCUL,MX, } \\
\text { PASBTS,BC,PAS, } \\
\text { BTPBC,BTC, } \\
\text { BE,CUL }\end{array}$ & $1,2,3,4,5,6$ & & & \\
\hline $\begin{array}{l}\text { Oryzomys couesi couesi } \\
\quad \text { (Alston, 1877) }\end{array}$ & $\begin{array}{l}\text { PCG,SMO,SMOC, } \\
\text { SMS,VC }\end{array}$ & $1,8,10,11,13,14,26,27$ & & $1,2,3,4,5$ & & & \\
\hline $\begin{array}{l}\text { Oryzomys fulgens Thomas, } \\
189 \mathrm{MX}\end{array}$ & $\begin{array}{l}\text { FT,MVC,MVO,PCT, } \\
\text { PCP,SMO, } \\
\text { SMS,VC }\end{array}$ & $\begin{array}{l}1,4,6,9,10,11 \\
13,17,18 \\
21,22,25,29\end{array}$ & & $1,2,3,4,5,6$ & & & \\
\hline $\begin{array}{l}\text { Oryzomys fulgens aztecus } \\
\text { Merriam, } 190 \mathrm{MX}\end{array}$ & FT,SMO,VC & $1,4,6,22$ & BTC,BTP & $2,3,4,5$ & & & \\
\hline $\begin{array}{l}\text { Oryzomys fulgens mexicanus } \\
\text { J. A. Allen, } 1897\end{array}$ & $\begin{array}{l}\text { MVC,MVO,PCT, } \\
\text { PCP,SMO,SMS }\end{array}$ & $\begin{array}{l}9,10,11,13 \\
17,18,21,25,29\end{array}$ & & $1,2,3,4,5,6$ & & & \\
\hline $\begin{array}{l}\text { Oryzomys melanotis Thomas, } \\
189 \mathrm{MX}\end{array}$ & & & & & & & \\
\hline $\begin{array}{l}\text { Oryzomys melanotis } \\
\quad \text { melanotis Thomas, } 189 \mathrm{MX} \\
\text { Oryzomys rostratus Merriam, } \\
1901\end{array}$ & PCP,SMS & 9,11 & ВTC,BTP & 2 & & & \\
\hline $\begin{array}{l}\text { Oryzomys rostratus rostratus } \\
\text { Merriam, } 190 \mathrm{MX}\end{array}$ & $\begin{array}{l}\text { MVC,MVO,PCG, } \\
\text { PCP,SMO,SMOC, } \\
\text { SMS }\end{array}$ & $\begin{array}{l}2,8,10,17 \\
24,25,26,27\end{array}$ & BTS,CAFBC,BMM & $1,2,3,4,5,6$ & & & \\
\hline $\begin{array}{l}\text { Rheomys mexicanus } \\
\text { Goodwin, } 1959 \mathrm{OAX}\end{array}$ & MVC,PCT,SMO & $8,10,13$ & BC,CUL & 1,4 & $\operatorname{Pr}$ & EN & \\
\hline $\begin{array}{l}\text { Sigmodon alleni Bailey, } 190 \\
\text { XX }\end{array}$ & $\begin{array}{l}\text { MVC,PCG,PCT, } \\
\text { SMO,SMS }\end{array}$ & $\begin{array}{l}11,13,17 \\
21,26,28\end{array}$ & $\begin{array}{l}\text { BC,BMM, } \\
\text { BTC,BTS }\end{array}$ & $1,2,3,4,5$ & & VU & \\
\hline $\begin{array}{l}\text { Sigmodon leucotis Bailey, } \\
190 \text { MX }\end{array}$ & & & & & & & \\
\hline $\begin{array}{l}\text { Sigmodon leucotis alticola } \\
\text { Bailey, } 190 \mathrm{MX}\end{array}$ & SMS, VC & $1,13,29$ & $\mathrm{BC}$ & 4,5 & & & \\
\hline $\begin{array}{l}\text { Sigmodon mascotensis J. A. } \\
\quad \text { Allen, } 189 \mathrm{MX}\end{array}$ & $\begin{array}{l}\text { DIT,FT,MVC,MVO, } \\
\text { PCT,PCP,SMO, } \\
\text { SMS,VC }\end{array}$ & $\begin{array}{l}1,8,9,10,13 \\
17,18,20,21 \\
22,24,28\end{array}$ & $\begin{array}{l}\text { BC,BTC,BE,BTS, } \\
\text { BTP,CUL,VS }\end{array}$ & $1,2,3,4,5$ & & & \\
\hline
\end{tabular}




\begin{tabular}{|c|c|c|c|c|c|c|c|}
\hline Lista sistemática & Subprovincia fisiográfica & Distrito & Vegetación & Altitud & $\mathrm{NOM}$ & IUCN & Cites \\
\hline $\begin{array}{l}\text { Sigmodon toltecus (De } \\
\quad \text { Saussure, 1860) }\end{array}$ & $\begin{array}{l}\text { DIT,FT,MVC,MVO, } \\
\text { PCG,PCT,PCP, } \\
\text { SMO,SMOC,SMS,VC } \\
\text { FT, } \\
\text { MVO,PCG, } \\
\text { SMO,SMOC }\end{array}$ & $\begin{array}{l}1,4,7,8,9,10,11, \\
13,14,17,18,21, \\
22,23,24,26,27, \\
28,293,4,8,10,22,26\end{array}$ & $\begin{array}{l}\text { BTS,BC,BTP, } \\
\text { BMM,BTC, } \\
\text { MX,CUL, } \\
\text { VS BTS, } \\
\text { BC,BTP,BMM, } \\
\text { BTC,MX, } \\
\text { CUL,VS }\end{array}$ & $1,2,3,4,5,6$ & & & \\
\hline $\begin{array}{l}\text { Sigmodon planifrons Nelson } \\
\text { y Goldman, } 1933\end{array}$ & MVO, DIT & 10 & & 1,2 & & & \\
\hline $\begin{array}{l}\text { Nyctomys sumichrasti (De } \\
\text { Saussure, 1860) }\end{array}$ & $\begin{array}{l}\text { MVC,PCG,PCT,PCP, } \\
\text { SMO,SMOC,SMS }\end{array}$ & $\begin{array}{l}8,10,11,13,14 \\
17,21,22,26\end{array}$ & $\begin{array}{l}\text { BC,BTC,CAFBC, } \\
\text { BTC,BTS,CUL, } \\
\text { VSBS,BTP }\end{array}$ & $1,2,3,4,5,6$ & & & \\
\hline $\begin{array}{l}\text { Nyctomys sumichrasti } \\
\quad \text { colimensis Laurie, } 195 \text { MX }\end{array}$ & SMO,SMS & 8,13 & & 4,5 & & & \\
\hline $\begin{array}{l}\text { Nyctomys sumichrasti } \\
\text { pallidulus Goldman, } 193 \\
\text { MX }\end{array}$ & $\begin{array}{l}\text { MVC,PCG,PCT,PCP, } \\
\text { SMO,SMOC,SMS }\end{array}$ & $\begin{array}{l}10,11,13 \\
17,21,26\end{array}$ & & $1,2,3,4,5$ & & & \\
\hline $\begin{array}{l}\text { Nyctomys sumichrasti } \\
\text { sumichrasti (De Saussure, } \\
\text { 1860) }\end{array}$ & $\begin{array}{l}\text { PCG,PCT,SMO, } \\
\text { SMOC }\end{array}$ & $8,10,14,22,26$ & & $1,3,4,5,6$ & & & \\
\hline $\begin{array}{l}\text { Tylomys nudicaudus (Peters, } \\
\text { 1866) }\end{array}$ & $\begin{array}{l}\text { MVC,MVO,PCG, } \\
\text { PCP,SMO,SMOC, } \\
\text { SMS }\end{array}$ & $\begin{array}{l}8,9,10,11,14 \\
17,18,20,21 \\
25,26,28\end{array}$ & $\begin{array}{l}\text { BTS,CAF,BCBTP, } \\
\text { BTS,CUL }\end{array}$ & $1,2,3,4,5$ & & & \\
\hline $\begin{array}{l}\text { Tylomys nudicaudus } \\
\text { gymnurus Villa, } 194 \text { MX }\end{array}$ & MVC,PCG,PCP,SMO & $8,10,14,21,26,28$ & & $1,2,3,4,5$ & & & \\
\hline $\begin{array}{l}\text { Tylomys nudicaudus } \\
\text { microdon Goodwin, } 1955 \\
\text { OAX }\end{array}$ & SMO.SMOC & 8,10 & & 1,2 & & & \\
\hline $\begin{array}{l}\text { Tylomys nudicaudus villai } \\
\text { Schaldach, } 1966 \text { OAX }\end{array}$ & MVO & 20,25 & & 3,4 & & & \\
\hline \multicolumn{8}{|l|}{ Orden Carnívora } \\
\hline $\begin{array}{l}\text { Herpailurus yagouaroundi } \\
\text { (E. Geoffroy Saint-Hilaire, } \\
\text { 1803) }\end{array}$ & & & & & A & & I \\
\hline $\begin{array}{l}\text { Herpailurus yagouaroundi } \\
\text { fossata (Mearns, 1901) }\end{array}$ & $\begin{array}{l}\text { MVO,PCG,PCT, } \\
\text { PCP,SMO,SMOC,SMS }\end{array}$ & $\begin{array}{l}7,8,9,10,11 \\
17,21,26,27\end{array}$ & $\begin{array}{l}\text { BC,BTP,BTC, } \\
\text { BTS,VS, }\end{array}$ & $1,2,3,4,5,6$ & & & \\
\hline $\begin{array}{r}\text { Leopardus pardalis } \\
\text { (Linnaeus, } 1758)\end{array}$ & $\begin{array}{l}\text { PCG,PCT,PCP, } \\
\text { SMOC,SMS }\end{array}$ & $9,10,17,21,26$ & $\begin{array}{l}\text { BTC,BTP,BTS, } \\
\text { CAF,VS }\end{array}$ & 1,2 & $\mathrm{P}$ & & I \\
\hline $\begin{array}{l}\text { Leopardus pardalis nelsoni } \\
\quad \text { (Goldman, 1925) }\end{array}$ & PCP,SMS & 17,21 & BTC,BTP,BTS,CAF,VS & 1 & & & \\
\hline $\begin{array}{l}\text { Leopardus pardalis pardalis } \\
\quad \text { (Linnaeus, 1758) }\end{array}$ & PCT, PCP & 10,21 & BTC,BTS & 1 & & & \\
\hline $\begin{array}{l}\text { Leopardus wiedii (Schinz, } \\
\text { 1821) }\end{array}$ & $\begin{array}{l}\text { FT,MVO,PCG,PCP, } \\
\text { PCT,SMO,SMOC, } \\
\text { SMS,VC }\end{array}$ & $\begin{array}{l}1,4,8,9,10,11,17 \\
21,22,23,26,27\end{array}$ & $\begin{array}{l}\text { BC,BMM,BTP,BTC, } \\
\text { BTS,CAF }\end{array}$ & $1,2,3,4,5,6$ & $\mathrm{P}$ & NT & I \\
\hline $\begin{array}{l}\text { Leopardus wiedii oaxacensis } \\
\text { (Nelson y Goldman, 1931) }\end{array}$ & $\begin{array}{l}\text { MVO,PCT,PCP,SMO, } \\
\text { SMOC,SMS,VC }\end{array}$ & $\begin{array}{l}1,8,10,11,17 \\
21,22,23,26\end{array}$ & $\begin{array}{l}\text { BC,BMM,BTP, } \\
\text { BTC,BTS,CAF }\end{array}$ & $1,2,3,4$ & & & \\
\hline $\begin{array}{l}\text { Leopardus wiedii yucatanicus } \\
\text { (Nelson y Goldman, 1931) }\end{array}$ & SMO & 10 & BTP & 1 & & & \\
\hline Lynx rufus (Schreber, 1777) & $\begin{array}{l}\text { FT,MVO,MVC, } \\
\text { SMO,VC }\end{array}$ & $\begin{array}{l}1,4,7,8,21 \\
22,24,28\end{array}$ & $\begin{array}{l}\text { BC,BTS, } \\
\text { BTC,CUL }\end{array}$ & $1,2,4,6,5,7$ & & & II \\
\hline $\begin{array}{l}\text { Lynx rufus escuinapae J. A. } \\
\text { Allen, } 1903\end{array}$ & MVO & 7 & BTC & 4 & & & \\
\hline $\begin{array}{l}\text { Lynx rufus oaxacensis } \\
\text { Goodwin, } 1963^{\text {OAX }}\end{array}$ & MVC,SMO,VC & $1,8,21,24,28$ & BC,BTS,BTC,CUL & $1,2,4,5$ & & & \\
\hline $\begin{array}{l}\text { Puma concolor (Linnaeus, } \\
\text { 1771) }\end{array}$ & & & & & & & II \\
\hline $\begin{array}{l}\text { Puma concolor cougar (Kerr, } \\
\text { 1792) }\end{array}$ & $\begin{array}{l}\text { PCT,PCP,SMO, } \\
\text { SMOC,SMS }\end{array}$ & $8,9,10,21,26,27$ & BTC,BTS & $1,2,3,4,5,6$ & & & \\
\hline $\begin{array}{l}\text { Panthera onca* (Linnaeus, } \\
\text { 1758) }\end{array}$ & $\begin{array}{l}\text { DIT,FT,MVC,PCG, } \\
\text { PCT,PCP,SMO, } \\
\text { SMOC,SMS,VC }\end{array}$ & $\begin{array}{l}2,4,8,10,14,17 \\
21,22,24,26,27,28\end{array}$ & $\begin{array}{l}\text { BTS,CAF,CUL,BTP, } \\
\text { BMM,BTC,VSBTC, } \\
\text { BTS,BC,CUL }\end{array}$ & $1,2,3,4,5,6,7$ & $\mathrm{P}$ & NT & I \\
\hline
\end{tabular}




\begin{tabular}{|c|c|c|c|c|c|c|c|}
\hline Lista sistemática & Subprovincia fisiográfica & Distrito & Vegetación & Altitud & NOM & IUCN & Cites \\
\hline $\begin{array}{l}\text { Panthera onca hernandesii } \\
\text { (Gray, 1857) } \mathrm{MX}\end{array}$ & $\begin{array}{l}\text { DIT,MVC,PCT, } \\
\text { PCP,SMOC }\end{array}$ & $10,21,28$ & & 1,3 & & & \\
\hline $\begin{array}{l}\text { Panthera onca veraecrucis } \\
\text { (Nelson y Goldman, 1933) } \\
\text { MX }\end{array}$ & PCG,SMO & 26,27 & & 3,4 & & & \\
\hline \multicolumn{8}{|l|}{ Familia Canidae } \\
\hline Canis latrans Say, 1822 & $\begin{array}{l}\text { MVC,MVO,PCG, } \\
\text { PCT,PCP,SMO, } \\
\text { SMS,VC }\end{array}$ & $\begin{array}{l}1,7,8,9,10 \\
11,21,24,26,28\end{array}$ & BC,BTC,CUL & $\begin{array}{l}1,2,3,4 \\
5,6,7\end{array}$ & & & \\
\hline $\begin{array}{l}\text { Canis latrans cagottis } \\
\quad \text { (Hamilton Smith, 1839) }\end{array}$ & $\mathrm{MVO}, \mathrm{SMO}, \mathrm{VC}$ & $1,7,26$ & BC,CUL & $3,4,7$ & & & \\
\hline $\begin{array}{l}\text { Canis latrans goldmani } \\
\text { Merriam, } 1904\end{array}$ & PCT,PCP,SMS & $10,21,28$ & BTC,CUL & 1,5 & & & \\
\hline \multicolumn{8}{|l|}{ Canis lupus Linnaeus, 1758} \\
\hline $\begin{array}{l}\text { Canis lupus baileyi Nelson y } \\
\text { Goldman, } 1929\end{array}$ & MVC & 21 & BTC & 1 & $\mathrm{E}$ & EW & \\
\hline $\begin{array}{l}\text { Urocyon cinereoargenteus } \\
\quad \text { (Schreber, 1775) }\end{array}$ & $\begin{array}{l}\text { DIT,FT,MVC,MVO, } \\
\text { PCG,PCT,PCP, } \\
\text { SMO,SMOC,SMS,VC }\end{array}$ & $\begin{array}{l}1,4,7,8,9,10,11 \\
14,17,21,22,26 \\
27,28,30\end{array}$ & $\begin{array}{l}\text { BC,BMM,CUL, } \\
\text { BTC,BTS }\end{array}$ & $1,2,3,4,5,6$ & & & \\
\hline $\begin{array}{l}\text { Urocyon cinereoargenteus } \\
\text { nigrirostris (Lichtenstein, } \\
1850 \text { ) }\end{array}$ & MVO,PCP,SMS & 7,11 & $\mathrm{BC}$ & $1,3,4$ & & & \\
\hline $\begin{array}{l}\text { Urocyon cinereoargenteus } \\
\text { orinomus Goldman, } 1938\end{array}$ & $\begin{array}{l}\text { FT,MVC,PCT,PCP, } \\
\text { SMO,SMS,VC }\end{array}$ & $\begin{array}{l}1,4,8,10,11,14 \\
21,22,28\end{array}$ & $\begin{array}{l}\text { BC,BMM,CUL, } \\
\text { BTC,BTS }\end{array}$ & $1,2,4,5$ & & & \\
\hline \multicolumn{8}{|l|}{ Familia Mephitidae } \\
\hline \multicolumn{8}{|l|}{$\begin{array}{l}\text { Conepatus leuconotus } \\
\text { (Lichtenstein, 1832) }\end{array}$} \\
\hline $\begin{array}{l}\text { Conepatus leuconotus } \\
\text { leuconotus (Lichtenstein, } \\
1832 \text { ) }\end{array}$ & $\begin{array}{l}\text { FT,MVO,PCT,PCP, } \\
\text { SMO,SMS,VC }\end{array}$ & $\begin{array}{l}1,4,7,8,9,10,11 \\
17,21,24,27\end{array}$ & $\mathrm{BC}, \mathrm{BTC}, \mathrm{VS}$ & $1,2,3,4,5,6,7$ & & & \\
\hline \multicolumn{8}{|l|}{$\begin{array}{l}\text { Conepatus semistriatus } \\
\text { (Boddaert, 1785) }\end{array}$} \\
\hline $\begin{array}{l}\text { Conepatus semistriatus } \\
\text { amazonicus (Lichtenstein, } \\
\text { 1838) }\end{array}$ & SMOC & 10 & BTS & 1 & $\operatorname{Pr}$ & & \\
\hline \multicolumn{8}{|l|}{ Mephitis macroura } \\
\hline $\begin{array}{l}\text { Mephitis macroura macroura } \\
\text { Lichtenstein, } 1832\end{array}$ & $\begin{array}{l}\text { FT,MVC,MVO,PCG, } \\
\text { PCT,PCP,SMO, } \\
\text { SMOC,SMS,VC }\end{array}$ & $\begin{array}{l}1,4,7,8,9,10,14 \\
17,21,22,24 \\
26,29\end{array}$ & $\begin{array}{l}\text { BC,DC,CUL,MX, } \\
\text { BTC,BTS }\end{array}$ & $1,2,3,4,5,6$ & & & \\
\hline \multicolumn{8}{|l|}{$\begin{array}{l}\text { Spilogale angustifrons A. H. } \\
\text { Howell, } 1902\end{array}$} \\
\hline $\begin{array}{l}\text { Spilogale angustifrons } \\
\text { tropicalis A. H. Howell, } \\
1902\end{array}$ & $\begin{array}{l}\text { MVC,MVO,PCT, } \\
\text { PCP,SMO,SMS,VC }\end{array}$ & $\begin{array}{l}1,7,8,10 \\
21,28\end{array}$ & $\begin{array}{l}\text { BC,CUL, } \\
\text { BTC,BTS }\end{array}$ & $1,2,3,4,6,7$ & & & \\
\hline $\begin{array}{l}\text { Spilogale pygmaea Thomas, } \\
189 \text { MX }\end{array}$ & & & & & A & $\mathrm{VU}$ & \\
\hline $\begin{array}{l}\text { Spilogale pygmaea australis } \\
\text { Hall, } 1938\end{array}$ & PCT,PCP,SMS & $\begin{array}{l}1,9,10 \\
11,17,21\end{array}$ & BMM,BTC,CUL & 1,4 & & & \\
\hline \multicolumn{8}{|l|}{ Familia Mustelidae } \\
\hline $\begin{array}{l}\text { Lontra longicaudis* (Olfers, } \\
\text { 1818) }\end{array}$ & & & & & A & & \\
\hline $\begin{array}{l}\text { Lontra longicaudis annectens } \\
\quad \text { (Major, 1897) }\end{array}$ & $\begin{array}{l}\text { FT,PCP,SMO, } \\
\text { SMOC,SMS,PCG }\end{array}$ & $\begin{array}{l}4,8,10,11 \\
17,18,21\end{array}$ & & $1,2,6$ & & I & \\
\hline $\begin{array}{l}\text { Eira barbara (Linnaeus, } \\
1758)\end{array}$ & & & & $1,2,3,4,6$ & $\mathrm{P}$ & & \\
\hline $\begin{array}{l}\text { Eira barbara senex (Thomas, } \\
\text { 1900) }\end{array}$ & $\begin{array}{l}\text { MVC,PCG,PCT, } \\
\text { SMO,SMOC }\end{array}$ & $\begin{array}{l}8,10,14,21 \\
22,26,27\end{array}$ & $\mathrm{BM}, \mathrm{BTS}, \mathrm{BTP}$ & & & & \\
\hline $\begin{array}{l}\text { Galictis vittata (Schreber, } \\
\text { 1776) }\end{array}$ & & & & & A & & \\
\hline $\begin{array}{l}\text { Galictis vittata canaster } \\
\quad \text { Nelson, } 1901\end{array}$ & DIT & 10 & BTP & 1 & & & \\
\hline
\end{tabular}




\begin{tabular}{|c|c|c|c|c|c|c|c|}
\hline Lista sistemática & Subprovincia fisiográfica & Distrito & Vegetación & Altitud & NOM & IUCN & Cites \\
\hline $\begin{array}{l}\text { Mustela frenata Lichtenstein, } \\
1831\end{array}$ & $\begin{array}{l}\text { MVC,MVO,PCG,PCP, } \\
\text { PCT,SMO,SMS,VC }\end{array}$ & $\begin{array}{l}1,8,9,13,14,17 \\
20,21,26,27,28\end{array}$ & $\begin{array}{l}\text { BC,BTC,BE, } \\
\text { BTS,CUL }\end{array}$ & $1,3,4,5,6$ & & & \\
\hline $\begin{array}{l}\text { Mustela frenata leucoparia } \\
\text { (Merriam, 1896) }\end{array}$ & MVC,MVO,SMS & $13,20,28$ & BC,CUL & 4,5 & & & \\
\hline $\begin{array}{l}\text { Mustela frenata } \\
\text { macrophonius (Elliot, } \\
\text { 1905) }\end{array}$ & $\begin{array}{l}\text { PCT,PCP, } \\
\text { SMO,SMS }\end{array}$ & $14,17,21$ & BTC,BE,BTS,CUL & 1,5 & & & \\
\hline $\begin{array}{l}\text { Mustela frenata perotae Hall, } \\
1936\end{array}$ & $\mathrm{SMO}, \mathrm{VC}$ & 1,8 & BC,CUL & 4,6 & & & \\
\hline \multicolumn{8}{|l|}{ Familia Procyonidae } \\
\hline $\begin{array}{l}\text { Bassariscus astutus } \\
\quad \text { (Lichtenstein, 1830) }\end{array}$ & $\begin{array}{l}\text { DIT,FT,MVC,MVO, } \\
\text { PCT,PCP,SMO,SMS }\end{array}$ & $\begin{array}{l}1,3,4,7,8,10 \\
14,21,22,27\end{array}$ & $\begin{array}{l}\text { BC,BMM,BTSBC, } \\
\text { BTC,BTS,CUL }\end{array}$ & $\begin{array}{l}1,2,3,4 \\
5,6,7\end{array}$ & & & \\
\hline $\begin{array}{l}\text { Bassariscus astutus astutus } \\
\quad \text { (Lichtenstein, 1830) }\end{array}$ & FT,MVO,SMO & $1,4,7,14,22$ & $\begin{array}{l}\text { BMM,BTSBC, } \\
\text { BTC,BTS,CUL }\end{array}$ & $2,3,4,5,7$ & & & \\
\hline $\begin{array}{l}\text { Bassariscus astutus } \\
\text { macdougalli Goodwin, } \\
1956 \text { OAX }\end{array}$ & $\begin{array}{l}\text { MVC,MVO,PCT, } \\
\text { PCP,SMS }\end{array}$ & 3,21 & BC,BTC,BTS,CUL & $1,3,4,5$ & & & \\
\hline $\begin{array}{l}\text { Bassariscus sumichrasti (De } \\
\quad \text { Saussure, 1860) }\end{array}$ & $\begin{array}{l}\text { MVO,PCT,PCP, } \\
\text { SMO,SMOC,SMS }\end{array}$ & $\begin{array}{l}8,10,11,17 \\
20,21\end{array}$ & $\begin{array}{l}\text { BMM,BTC,BTP, } \\
\text { BTS,CUL }\end{array}$ & $1,2,3,4$ & $\operatorname{Pr}$ & & \\
\hline $\begin{array}{l}\text { Bassariscus sumichrasti } \\
\text { oaxacensis (Goodwin, } \\
\text { 1956) }\end{array}$ & MVC,SMO,SMOC & 10,21 & BTP,BTS & 1,2 & & & \\
\hline $\begin{array}{l}\text { Bassariscus sumichrasti } \\
\text { sumichrasti (De Saussure, } \\
\text { 1860) }\end{array}$ & $\begin{array}{l}\text { PCT,PCP,SMO, } \\
\text { SMOC,SMS }\end{array}$ & $8,10,21$ & $\begin{array}{l}\text { BMM,BTC, } \\
\text { BTS,CUL }\end{array}$ & $1,2,3,4$ & & & \\
\hline $\begin{array}{l}\text { Bassariscus sumichrasti } \\
\quad \text { variabilis (Peters, 1874) }\end{array}$ & PCP,SMS & 11,17 & BTS,CAF & 2,4 & & & \\
\hline Potos flavus (Schreber, 1774) & & & & & $\operatorname{Pr}$ & & \\
\hline $\begin{array}{l}\text { Potos flavus chiriquensis J. A. } \\
\quad \text { Allen, } 1904\end{array}$ & $\begin{array}{l}\text { MVC,MVO,PCG, } \\
\text { PCT,PCP,SMO, } \\
\text { SMOC,SMS }\end{array}$ & $\begin{array}{l}8,9,10,11,17 \\
21,22,23,27\end{array}$ & $\begin{array}{l}\text { BC,BTC,BTS, } \\
\text { DC,CAF,CUL }\end{array}$ & $1,2,3,4,6$ & & & \\
\hline $\begin{array}{l}\text { Nasua narica (Linnaeus, } \\
\text { 1766) }\end{array}$ & $\begin{array}{l}\text { DIT,FT,MVC,MVO, } \\
\text { PCG,PCT,PCP,SMO, } \\
\text { SMOC,SMS }\end{array}$ & $\begin{array}{l}4,7,8,9,10,11,17 \\
21,22,26,27\end{array}$ & $\begin{array}{l}\text { BC,BTC,BTS,CAF, } \\
\text { CUL,MX,VS }\end{array}$ & $1,2,3,4,5,6$ & & & \\
\hline $\begin{array}{l}\text { Nasua narica molaris } \\
\quad \text { Merriam, } 1902\end{array}$ & MVO,PCP,SMS & $7,11,17,21$ & BTS,BTC,CAF,VS & $1,2,4$ & & & \\
\hline $\begin{array}{l}\text { Nasua narica narica } \\
\text { (Linnaeus, 1766) }\end{array}$ & $\begin{array}{l}\text { DIT,FT,MVC,PCG, } \\
\text { PCT,PCP,SMO, } \\
\text { SMOC,SMS }\end{array}$ & $\begin{array}{l}4,10,11,17 \\
21,22,26\end{array}$ & $\begin{array}{l}\text { BC,BTS,BTC, } \\
\text { CUL,MX }\end{array}$ & $1,2,3,4$ & & & \\
\hline \multicolumn{8}{|l|}{$\begin{array}{l}\text { Procyon lotor (Linnaeus, } \\
\text { 1758) }\end{array}$} \\
\hline $\begin{array}{l}\text { Procyon lotor hernandezii J. } \\
\text { A. Wagler, } 1831\end{array}$ & $\begin{array}{l}\text { DIT,FT,MVC,MVO, } \\
\text { PCG,PCT,PCP, } \\
\text { SMO,SMOC,SMS }\end{array}$ & $\begin{array}{l}4,7,8,9,10,11,13 \\
17,20,21,22 \\
26,27,30\end{array}$ & $\begin{array}{l}\text { BTS,BTC,DC,MXBC, } \\
\text { CUL,BTC,MX,BE,BTS }\end{array}$ & $1,2,3,4,5,6$ & & & \\
\hline \multicolumn{8}{|l|}{ Orden Artiodactyla } \\
\hline $\begin{array}{l}\text { Dicotyles angulatus Cope, } \\
1889\end{array}$ & $\begin{array}{l}\text { DIT,MVC,PCG,PCT, } \\
\text { PCP,SMO,SMOC,SMS, } \\
\text { VC PCG,PCT }\end{array}$ & $\begin{array}{l}1,6,8,9,10,11 \\
17,21,26,27\end{array}$ & $\begin{array}{l}\text { BTP,BTS,BTC, } \\
\text { BE,CAF }\end{array}$ & $1,2,3,4,6$ & & & \\
\hline Tayassu pecari (Link, 1795) & & & & & $\mathrm{P}$ & VU & \\
\hline $\begin{array}{l}\text { Tayassu pecari ringens* } \\
\text { Merriam, } 1901\end{array}$ & PCT,SMOC & 10 & BTS,BTC,CAF,VS & 1,3 & & & \\
\hline \multicolumn{8}{|l|}{ Familia Cervidae } \\
\hline Mazama temama (Kerr, 1792) & & & & & & & \\
\hline $\begin{array}{l}\text { Mazama temama temama* } \\
\quad \text { (Kerr, 1792) }\end{array}$ & $\begin{array}{l}\text { DIT,PCG,PCT,PCP, } \\
\text { SMO,SMOC,SMS }\end{array}$ & $\begin{array}{l}4,8,10,17 \\
21,22,26,27\end{array}$ & BTP,BTS,BTC,CUL & $1,2,3,4,6$ & & & \\
\hline $\begin{array}{l}\text { Odocoileus virginianus* } \\
\quad(\text { Zimmermann, } 1780)\end{array}$ & $\begin{array}{l}\text { DB,DIT,FT,MVC, } \\
\text { MVO,PCT,PCP,SMO, } \\
\text { SMS,SMOC,VC }\end{array}$ & $\begin{array}{l}1,4,6,7,8,9,10 \\
11,13,17,18,21,22 \\
24,27,28,29\end{array}$ & $\begin{array}{l}\text { BC,BMM,BTS,BTC, } \\
\text { BTP,CAF,MX,VS,CUL }\end{array}$ & $1,2,3,4,5,6,7$ & & & \\
\hline $\begin{array}{l}\text { Odocoileus virginianus } \\
\quad \text { acapulcensis (Caton, 1877) }\end{array}$ & PCT,PCP,SMS & $17,21,28$ & $\begin{array}{l}\text { BTS,BTC,CAF, } \\
\text { BC,VS,CUL }\end{array}$ & $1,2,3$ & & & \\
\hline $\begin{array}{l}\text { Odocoileus virginianus } \\
\text { mexicanus (Gmelin, 1788) }\end{array}$ & MVO & 7 & $\mathrm{BE}$ & 4 & & & \\
\hline
\end{tabular}




\begin{tabular}{|c|c|c|c|c|c|c|c|}
\hline Lista sistemática & Subprovincia fisiográfica & Distrito & Vegetación & Altitud & $\mathrm{NOM}$ & IUCN & Cites \\
\hline $\begin{array}{l}\text { Odocoileus virginianus } \\
\text { oaxacensis Goldman y } \\
\text { Kellogg, } 1940 \text { OAX }\end{array}$ & PCT,SMO,VC & $\begin{array}{l}1,8,10,22 \\
24,27,29\end{array}$ & BC,BTP,BTS,BMM & $1,2,3,4,5,6,7$ & & & \\
\hline $\begin{array}{l}\text { Odocoileus virginianus } \\
\text { thomasi Merriam, } 1898\end{array}$ & $\begin{array}{l}\text { DIT,FT,PCT,SMO, } \\
\text { SMOC }\end{array}$ & $6,10,21,22$ & $\begin{array}{l}\text { BTC,MX,BTP, } \\
\text { BTS,CUL }\end{array}$ & $1,2,3$ & & & \\
\hline $\begin{array}{l}\text { Orden Perissodactyla } \\
\text { Familia Tapiridae }\end{array}$ & & & & & & & \\
\hline Tapirella bairdii* (Gill, 1865) & $\begin{array}{l}\text { PCG,PCT,PCP, } \\
\text { SMO,SMOC }\end{array}$ & $2,8,9,10,21,27$ & BMM,ВTC,BTS,ВTP & $1,2,3,4$ & $\mathrm{P}$ & EN & I \\
\hline
\end{tabular}

\section{Referencias}

Alfaro, A. M., García-García, J. L. y Santos-Moreno, A. (2005). The false vampiro bat, Vampyrum spectrum, in Oaxaca, Mexico. Bats Research News, 46, $145-146$.

Alfaro, A. M. y Santos-Moreno, A. (2012). The big free-tailed bat Nyctinomops macrotis (Chiroptera: Molossidae) in Oaxaca, Mexico. Chiroptera Neotropical, 18, 1115-1116.

Ávila-Valle, Z. A., Castro-Campillo, A., León-Paniagua, L., Salgado-Ugalde, I. H., Navarro-Sigüenza, A. G., Hernández-Baños, B. E., et al. (2012). Geographic variation and molecular evidence of the Blackish Deer Mouse complex (Peromyscus furvus Rodentia: Muridae). Mammalian Biology, 77, 166-177.

Bacaro, G., Ricotta, C. y Mazzoleni, S. (2007). Measuring beta-diversity from taxonomic similarity. Journal of Vegetation Science, 18, 793-798.

Botello, F., Illoldi-Rangel, P., Linaje, M. y Sánchez-Cordero, V. (2007). New record of the rock squirrel (Spermophilus variegatus) in the state of Oaxaca. Mexico. The Southwestern Naturalist, 52, 326-328.

Briones-Salas, M. A., Cortés-Marcial, M. y Bonilla, C. (2006). Colección regional mastozoológica de Oaxaca. En C. Lorenzo-Monterrubio, E. Espinoza-Medinilla, M. A. Briones-Salas y F. Cervantes (Eds.), Colecciones mastozoológicas de México (pp. 447-468). México, D.F.: Instituto de Biología, Universidad Nacional Autónoma de México-Asociación Mexicana de Mastozoología, A.C.

Briones-Salas, M., Peralta-Pérez, M. y García-Luis, M. (2013). Acoustic characterization of new species of bats for the State of Oaxaca. Mexico. Therya, $4,15-32$.

Briones-Salas, M. A. y Sánchez-Cordero, V. (2004). Mamíferos. En A. J. García-Mendoza, M. J. Ordóñez y M. Briones-Salas (Eds.), Biodiversidad de Oaxaca (pp. 423-447). México, D.F.: Instituto de Biología, Universidad Nacional Autónoma de México-Fondo Oaxaqueño para la Conservación de la Naturaleza-World Wildlife Found.

Carraway, L. N. (2007). Shrews (Eulypotyphla: Soricidae) of Mexico. Monographs of the Western North American Naturalist, 3, 1-91.

Ceballos, G. y Oliva, G. (2005). Los mamíferos silvestres de México. México, D.F.: Comisión Nacional para el Conocimiento y Uso de la BiodiversidadFondo de Cultura Económica.

CITES (Convención sobre el Comercio Internacional de Especies Amenazadas de Fauna y Flora Silvestres). (2011). Appendices I, II, and III. Convention on international trade in endangered species of wild fauna and flora. Ginebra: United Nations Environment Programme.

Escalante, T. (2003). Determinación de prioridades en las áreas de conservación para los mamíferos terrestres de México, empleando criterios biogeográficos. Anales del Instituto de Biología, Universidad Nacional Autónoma de México, Serie Zoología, 74, 211-237.

García-García, J. L., Santos-Moreno, A., Alfaro, A. M. y Soto-Centeno, A. (2007). Noteworthy records of Eptesicus brasiliensis (Vespertilionidae) from Oaxaca, Mexico. Bats Research News, 48, 5-6.

García-Grajales, J. y Buenrostro, A. (2012). Revisión al conocimiento de los murciélagos del estado de Oaxaca. Therya, 3, 277-293.

García-Marmolejo, G., Escalante, T. y Morrone, J. J. (2008). Establecimiento de prioridades para la conservación de mamíferos terrestres neotropicales de México. Mastozoología Neotropical, 15, 41-65.
García-Mendoza, A. J. (2004). Integración del conocimiento florístico del estado. En A. J. García-Mendoza, M. J. Ordóñez y M. Briones-Salas (Eds.), Biodiversidad de Oaxaca (pp. 305-325). México, D.F.: Instituto de Biología, Universidad Nacional Autónoma de México-Fondo Oaxaqueño para la Conservación de la Naturaleza-World Wildlife Found.

González-Pérez, G., Briones-Salas, M. y Alfaro, A. M. (2004). Integración del conocimiento faunístico del estado. En A. J. García-Mendoza, M. J. Ordóñez y M. Briones-Salas (Eds.), Biodiversidad de Oaxaca. (pp. 349-366). México, D.F.: Instituto de Biología, Universidad Nacional Autónoma de MéxicoFondo Oaxaqueño para la Conservación de la Naturaleza-World Wildlife Found.

Goodwin, G. G. (1969). Mammals from the state of Oaxaca, Mexico, in the American Museum of Natural History. Bulletin American Museum of Natural History, 141, 1-269.

Guevara-Chumacero, L. M., López-Wilchis, R. y Sánchez-Cordero, V. (2001). 105 años de investigación mastozoológica en México (1890-1995): una revisión de sus enfoques y tendencias. Acta Zoológica Mexicana (nueva serie), $83,35-72$.

Halffter, G., Llorente-Bousquets, J. y Morrone, J. J. (2008). La perspectiva biogeográfica histórica. En J. Soberón, G. Halffter y J. Llorente-Bousquets (Eds.), Capital natural de México, Vol. I. Conocimiento actual de la biodiversidad (pp. 67-86). México, D.F.: Comisión Nacional para el Conocimiento y Uso de la Biodiversidad.

Hall, E. R. (1981). The mammals of North America. New York: John Wiley and Sons.

IUCN (International Union for Conservation of Nature). (2014). IUCN Red list of threatened species. International Union for Conservancy of Nature and Natural Resources. Recuperado el 15 enero 2010 de http://www.iucnredlist.org/

Izsák, C. y Price, R. G. (2001). Measuring b-diversity using a taxonomic similarity index, and its relation to spatial scale. Marine Ecology, 215, 69-77.

Koleff, P. y Soberón, J. (2008). Patrones de diversidad espacial en grupos selectos de especies. En J. Soberón, G. Halffter y J. Llorente-Bousquets (Eds.), Capital natural de México, Vol. I. Conocimiento actual de la biodiversidad. (pp. 323-365). México, D.F.: Comisión Nacional para el Conocimiento y Uso de la Biodiversidad.

León-Paniagua, A., Navarro-Sigüenza, A. G., Hernández-Baños, B. E. y Morales, J. C. (2007). Diversification of the arboreal mice of the genus Habromys (Rodentia: Cricetidae: Neotominae) in the Mesoamerican highlands. Molecular Phylogenetics and Evolution, 42, 653-664.

Lira, I. y Sánchez-Cordero, V. (2006). Nuevo registro de Conepatus semistriatus Boddaert. 1784 (Carnívora: Mustelidae) en Oaxaca, México. Acta Zoológica Mexicana (nueva serie), 22, 119-121.

Llorente, J. y Ocegueda, S. (2008). Estado del conocimiento de la biota. En J. Soberón, G. Halffter y J. Llorente-Bousquets (Eds.), Capital natural de México, Vol. I. Conocimiento actual de la biodiversidad (pp. 283-322). México, D.F.: Comisión Nacional para el Conocimiento y Uso de la Biodiversidad.

Lorenzo, C., Álvarez-Castañeda, S. T., Arellano, E., Arroyo-Cabrales, J., Bolaños, J., Briones-Salas, M., et al. (2012). Los mamíferos de México en las colecciones científicas de Norteamérica. Therya, 3, 239-262.

Luna-Vega, I. (2008). Aplicaciones de la biogeografía histórica a la distribución de las plantas mexicanas. Revista Mexicana de Biodiversidad, 79, 217-241. 
Morrone, J. J. (2001). Biogeografía de América Latina y el Caribe. Zaragoza: M y T-Manuales y Tesis Sociedad Entomológica Aragonesa.

Olguín, H. C., León-Paniagua, L., Samper-Palacios, U. M. y Sánchez-Cordero, V. (2008). Mastofauna de la región de los Chimalapas, Oaxaca, México. En C. Lorenzo, E. Espinoza y J. Ortega (Eds.), Avances en el estudio de los mamíferos de México, Vol. II. México, D.F.: Publicaciones especiales. Asociación Mexicana de Mastozoología, A.C, pp. 165-126

Ortiz-Pérez, M. A., Hernández-Santana, J. R. y Figueroa-Mah-Eng, J. M. (2004). Reconocimiento fisiográfico y geomorfológico. En A. J. GarcíaMendoza, M. J. Ordóñez y M. Briones-Salas (Eds.), Biodiversidad de Oaxaca. (pp. 43-53). México, D.F.: Instituto de Biología, Universidad Nacional Autónoma de México-Fondo Oaxaqueño para la Conservación de la Naturaleza-World Wildlife Found.

Ramírez-Pulido, J., Briton, M., Perdomo, A. y Castro, A. (1986). Guía de los mamíferos de México. México D.F.: Universidad Autónoma Metropolitana.

Ramírez-Pulido, J., González-Ruíz, N., Gardner, A. L. y Arroyo-Cabrales, J. (2014). List of recent land mammals of Mexico, 2014. Special Publications Museum of Texas Tech University., 63.

Retana, O. y Lorenzo, C. (2002). Lista de los mamíferos terrestres de Chiapas: endemismo y estado de conservación. Acta Zoológica Mexicana (nueva serie), $85,25-49$

Rodríguez, P., Soberón, J. y Arita, H. (2003). El componente beta de la diversidad de mamíferos de México. Acta Zoológica Mexicana (nueva serie), 89, 241-259.

Sánchez-Cordero, V., Botello, F., Flores-Martínez, J. J., Gómez-Rodríguez, R. A., Guevara, L., Gutiérrez-Granados, G., et al. (2014). Biodiversidad de Chordata (Mammalia) en México. Revista Mexicana de Biodiversidad, 85 Supl., S496-S504.

Santos-Moreno, J. A. y Gallardo, L. (2014). Three new species in the state of Oaxaca, Mexico. Chiroptera Neotropical, 20, 1226-1229.

Santos-Moreno, J. A., García-Orozco, S. y Pérez-Cruz, E. E. (2010). Records of bats from Oaxaca, Mexico. The Southwestern Naturalist, 55, 454-456.

Semarnat (Secretaría de Medio Ambiente y Recursos Naturales). (2010). Norma Oficial Mexicana NOM-059-SEMARNAT-2010, Protección ambientalEspecies nativas de México de flora y fauna silvestres-Categorías de riesgo y especificaciones para su inclusión, exclusión o cambio-Lista de especies en riesgo. Diario Oficial de la Federación. 30 de diciembre de 2010, Segunda Sección. México, 2454, 1-77.

Semarnat (Secretaría de Medio Ambiente y Recursos Naturales). (2014). Norma Oficial Mexicana NOM-059-SEMARNAT-2010. Acuerdo por el que da a conocer la lista de especies y poblaciones prioritarias para la conservación. Diario Oficial de la Federación, 726, 1-14.

Sosa-Escalante, J., Hernández-Betancourt, S., Pech-Canche, J. M., MacSwinney, M. C. y Díaz-Gamboa, R. (2014). Los mamíferos del estado de Yucatán. Revista Mexicana de Mastozoología Nueva Época, 4, 1-20.

Suárez-Mota, M. E. y Villaseñor, J. L. (2011). Las compuestas endémicas de Oaxaca, México: diversidad y distribución. Boletín de la Sociedad Botánica de México, 88, 55-61.

Toledo, V. (1976). Los cambios climáticos del Pleistoceno y sus efectos sobre la vegetación tropical cálida y húmeda de México Tesis de Maestría. México, D.F.: Facultad de Ciencias, Universidad Nacional Autónoma de México.

Torres-Colín, R. (2004). Tipos de vegetación. En A. J. García-Mendoza, M. J. Ordóñez y M. Briones-Salas (Eds.), Biodiversidad de Oaxaca (pp. 105-117). México, D.F.: Instituto de Biología, Universidad Nacional Autónoma de México-Fondo Oaxaqueño para la Conservación de la Naturaleza-World Wildlife Found.

Torres-Morales, L., Rodríguez-Aguilar, G., Cabrera-Cruz, S. A. y VillegasPatraca, R. (2014). Primer registro de Eumops nanus (Chiroptera: Molossidae) en Oaxaca, México. Mastozoología Neotropical, 21, 373-378.

Trejo, I. (2004). Clima. En A. J. García-Mendoza, M. J. Ordóñez y M. BrionesSalas (Eds.), Biodiversidad de Oaxaca. (pp. 67-85). México, D.F.: Instituto de Biología, Universidad Nacional Autónoma de México-Fondo Oaxaqueño para la Conservación de la Naturaleza-World Wildlife Found.

Vallejo, R. M. y González-Cózatl, F. X. (2012). Phylogenetic affinities and species limits within the genus Megadontomys (Rodentia: Cricetidae) based on mitochondrial sequence data. Journal Zoological Systematics and Evolutionary Research, 50, 67-75.

Velázquez, A., Durán, E., Ramírez, I., Mas, J. F., Bocco, G. Ramírez, G. et al. (2003). Land use-cover change process in highly biodiverse areas: the case of Oaxaca, México. Global Environmental Change, 13, 157-184. 